\title{
Regiospecific synthesis of 5,7-disubstituted quinoxalino[2,3-b]phenazines
}

\author{
Panayiotis A. Koutentis \\ University of Cyprus, Department of Chemistry, P.O. Box 20537, 1678 Nicosia, Cyprus \\ E-mail: koutenti@ucy.ac.cy
}

\section{Dedicated to Prof. Charles W. Rees on the occasion of his $75^{\text {th }}$ birthday}

(received 14 Jul 02; accepted 04 Sep 02; published on the web 12 Sep 02)

\begin{abstract}
Hydrogenation of the readily prepared dinitrobenzenediamines 7 followed by air oxidation affords the green colored 5,7-disubstituted-5H,12H-quinoxalino[2,3- $b$ ]phenazines 3 in good yields. Mechanistic rationale, compound characterisation and full experimental details are provided.
\end{abstract}

Keywords: Heterocycles, zwitterions, fluorindine, quinoxalino[2,3-b]phenazines, tetraazapentacenes

\section{Introduction}

Interest in the heterocyclic system quinoxalino[2,3- $b]$ phenazine (Flourindine) has reemerged. Recently theoretical and experimental studies on the 5,7-diphenyl-5H,12H-quinoxalino[2,3$b]$ phenazine 3 established it has a singlet ground state resulting in a zwitterionic structure. ${ }^{1}$ A 5,7-bisoctadecyl derivative has shown interesting high temperature liquid crystalline properties. $^{2,3}$ These studies were made possible by an improved high yielding and regiospecific synthesis of the 5,7-disubstitued isomers. We now wish to report the full synthetic details. 
<smiles>c1ccc2nc3cc4nc5ccccc5nc4cc3nc2c1</smiles>

1<smiles>C1=CC2=Nc3ccccc3N(c3ccccc3)C2=CC(c2ccccc2)=C1</smiles>

3<smiles>c1ccc2c(c1)Nc1cc3nc4ccccc4nc3cc1N2</smiles>

2<smiles>C1=CC2N=c3cc4c(cc3N(c3ccccc3)C2C=C1)=Nc1ccccc1N4c1ccccc1</smiles>

The parent system quinoxalino[2,3- $b]$ phenazine $\mathbf{1}$ is not known. The only dihydroquinoxalino[2,3-b]phenazine is the $5 H, 14 H$-dihydro derivative 2 thought to be in equilibrium with the $5 H, 12 H$-dihydro isomer. ${ }^{4}$ However, 5,7-diphenyl-5H,12H-quinoxalino[2,3-b]-phenazine (diphenylisofluorindine, 5,7-DPQP, 3a) exists. ${ }^{5}$ The preparation of 5,7-DPQP from the treatment of 3-imino- $N, 5$-diphenyl-3H,5H-2-phenazinamine (3-anilinoaposafranine) with $\mathrm{N}$-phenyl-1,2benzenediamine 5 and two equivalents of mineral acid in refluxing benzoic acid was reported over a 100 years ago, however, at that time its electronic structure was not understood. ${ }^{5}$ The product which did not melt (up to $260{ }^{\circ} \mathrm{C}$ ) was identified by microanalysis and by comparison of its physical appearance and color in solution with its more commonly known isomer 5,12-diphenyl-5H,12H-quinoxalino[2,3- $b$ ]phenazine (diphenylfluorindine, 5,12-DPQP, 4). ${ }^{6}$ Both isomers dissolve in acid to give a blue solution with a red fluorescence but only the free base of 5,12-DPQP was observed to fluoresce strongly to the naked eye whilst that of the 5,7-DPQP did not. Both compounds crystallize to give blue-green crystals with a metallic luster. Various preparations of 5,12-DPQP are reported. ${ }^{6}$ In particular the treatment of 3-anilinoaposafranine with $N$-phenyl-1,2-benzenediamine 5 and one equivalent of mineral acid to give the isomer 5,12$\mathrm{DPQP}^{6}$ suggested to us that the formation of a mixture of both isomers was likely via this route and would therefore require separation. Furthermore the synthesis of 3-anilinoaposafranine was derived from the oxidative coupling of two equivalents of $N$-phenyl-1,2-benzenediamine 5 which gives a mixture of two isomeric products that again require careful separation. ${ }^{7}$

\section{Synthesis}

We proposed and successfully carried out a rational synthesis that affords 5,7-DPQP unambiguously and in good yield (Scheme 1). 
<smiles>[R]Nc1ccccc1N</smiles>

5a-d<smiles>O=[N+]([O-])c1cc([N+](=O)[O-])c(F)cc1F</smiles>

6

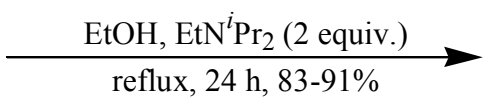

$\underset{\text { 1) } \mathrm{H}_{2}, \mathrm{Pd} / \mathrm{C}, \mathrm{EtOH}, 3 \mathrm{~h}, \mathrm{RT}}{\mathrm{EtOH} \text {, air, reflux, } 77-85 \%}$<smiles>[R]Nc1ccccc1Nc1cc(Nc2ccccc2N[R])c([N+](=O)[O-])cc1[N+](=O)[O-]</smiles>

7a-d<smiles></smiles>

3a-d

\section{Scheme 1}

1,5-Difluoro-2,4-dinitrobenzene 6 reacts with $N$-substituted-1,2-benzenediamines 5 to give dinitrobenzenediamines $\mathbf{7}$ in good yields. Hydrogenation of compounds $\mathbf{7}$ gave the benzenetetraamines $\mathbf{8}$, which on simple heating in ethanol in the presence of air gave the free base 3 . The benzenetetraamines 8 were very susceptible to oxidation and their isolation and characterization was only carried out with one example (c.f. compound 8a, Experimental section). Treatment of $\mathbf{8 a}$ with ethanol and hydrochloric acid gave the hydrochloride salt of 5,7DPQP which could be liberated with aqueous hydroxide.<smiles>[R]Nc1ccccc1Nc1cc(Nc2ccccc2N[R])c(N)cc1N</smiles>

8a, $\mathrm{R}=\mathrm{Ph}$
1) $\mathrm{EtOH}, \mathrm{HCl}$, reflux, $2 \mathrm{~h}$

2) $\mathrm{NaOH}(\mathrm{aq}), 91 \%$

3a

The $N$-aryl-1,2-benzenediamines 5 were prepared from 1-fluoro-2-nitrobenzene and anilines in the presence of potassium fluoride, ${ }^{8}$ followed by hydrogenation. The $N$-alkyl derivatives were prepared from the action of the more nucleophilic alkylamines on 1-fluoro-2-nitrobenzene in refluxing ethanol, followed by hydrogenation.

Unsymmetrical quinoxalino[2,3-b]phenazine 3e was prepared from the selective displacement of one fluoride from 1,5-difluoro-2,4-dinitrobenzene 6 which was achieved under mild conditions to give 5-fluoro-2,4-dinitrobenzamine 9 in good yield (Scheme 2). 
<smiles>[R]Nc1ccccc1N</smiles>

5a, $\mathrm{R}=\mathrm{Ph}$<smiles></smiles>

3e

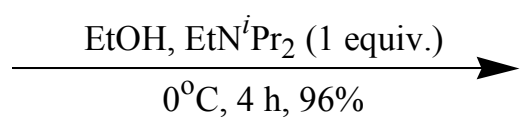

6<smiles>[R]Nc1ccccc1Nc1cc(F)c([N+](=O)[O-])cc1[N+](=O)[O-]</smiles>

9

EtOH, reflux $24 \mathrm{~h}, 91 \%$ $\frac{1 \text { 1) } \mathrm{H}_{2}, \mathrm{Pd} / \mathrm{C}, \mathrm{EtOH}, 3 \text { h, RT }}{\text { 2) } \mathrm{EtOH} \text {, air, reflux, } 58 \%}$

\section{Scheme 2}

Nearly quantitative yields (c.f. Method 1, compound 7a, Experimental section) were obtained for the preparation of compounds 7 with the use of 4 equivalents of benzenediamine 5 . The cost, however, of preparing more complex diamines 5 prevented the repeated use of 4 fold excesses and despite lower yields the use of 2 equivalents of diamine 5 followed by 2 equivalents of Hünig's base was preferred (c.f. Method 2, compound 7a, Experimental section). The overall synthesis, analogous to that used for the preparation of $5 H, 14 H$-quinoxalino[2,3- $b]$ phenazine from 1,5-dichloro-2,4-dinitrobenzene and excess 1,2-benzenediamine, ${ }^{9}$ allows the preparation of a variety of 5,7-disubstituted quinoxalinophenazines (Table 1).

Table 1. 5,7-Disubstituted quinoxalino[2,3-b]phenazines 3 and selected properties

\begin{tabular}{cccccc}
\hline Comp. No. & $\mathrm{R}$ & $\mathrm{R}^{\mid}$ & $\lambda_{\max } \mathrm{nm},(\log \varepsilon)^{a}$ & $\mathrm{mp}\left({ }^{\mathrm{o}} \mathrm{C}\right)^{b}$ & Yield (\%) \\
\hline 3a & $\mathrm{Ph}$ & $\mathrm{Ph}$ & $763(4.41)$ & $360-370$ dec. & $85-91$ \\
3b & $4-{ }^{n} \mathrm{BuC}_{6} \mathrm{H}_{4}$ & $4-{ }^{n} \mathrm{BuC}_{6} \mathrm{H}_{4}$ & $760(4.50)$ & $310-320$ dec. & 77 \\
3c & $4-{ }^{t} \mathrm{BuC}_{6} \mathrm{H}_{4}$ & $4-{ }^{t} \mathrm{BuC}_{6} \mathrm{H}_{4}$ & $763(4.35)$ & $350-360$ dec. & 84 \\
3d & ${ }^{n} \mathrm{Bu}$ & ${ }^{n} \mathrm{Bu}$ & $768(4.38)$ & $245-250$ dec. & 83 \\
3e & $4-{ }^{t} \mathrm{BuC}_{6} \mathrm{H}_{4}$ & $\mathrm{Ph}$ & $760(4.40)$ & $345-350$ dec. & 58 \\
\hline
\end{tabular}

${ }^{\mathrm{a}} \mathrm{UV} / \mathrm{VIS}$ recorded in dichloromethane, concentrations approximately 10-5 M. ${ }^{\mathrm{b}}$ Recrystallised from ethanol and dried overnight under vacuum $(30 \mathrm{mmHg})$ at $60 \mathrm{oC}$. 
Quinoxalinophenazine 3a is readily monomethylated in MeI to give the trisubstituted quinoxalinophenazine cation $\mathbf{1 0}$ that is dark blue in color and has a UV/vis spectrum $\left[\lambda_{\max } 652\right.$ $\mathrm{nm}(\log \varepsilon 4.56)]$ that closely resembles that of the monoprotonated 5,7- or 5,12-diphenyl quinoxalino[2,3- $b]$ phenazines. A bis protonated material $\mathbf{1 1}$ was crystallized from perchloric acid which exhibits a $\lambda_{\max }$ at 637 (5.01).<smiles></smiles><smiles>[Mg][Mg][Mg]</smiles>

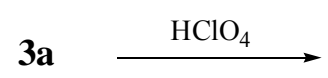<smiles></smiles>

Mechanistic rationale. The mechanism for the cyclization closely follows that proposed for the synthesis of dihydroquinoxalino[2,3-b] phenazine. ${ }^{9}$ A dilute solution of tetraamine 8 in DCM $\left(\lambda_{\max } 241 \mathrm{~nm}\right)$, at $c a .20 \mathrm{C}$, becomes brown in color (over $24 \mathrm{~h}$ ) and absorption spectroscopy shows the formation of two new strong absorptions at 283 and $472 \mathrm{~nm}$; the absorption at $241 \mathrm{~nm}$ is no longer visible. Over a period of 7 days the intensity of the absorption at $472 \mathrm{~nm}$ decreases until the spectrum resembles that of 5,7-DPQP; the solution's color changing from brown to green. This suggests that the first cyclization to give presumably phenazine $\mathbf{1 4}$ is more rapid than the second cyclization to give 5,7-DPQP 3a. A probable mechanism is described in Scheme 3. Air oxidation of benzenetetraamine 8a gives species 12 which can cyclize via nucleophilic attack of the diphenylamine on the NH imine to give $\mathbf{1 3}$ and ultimately quinoxalinophenazine $\mathbf{3 a}$. 
<smiles>Nc1cc(N)c(Nc2ccccc2Nc2ccccc2)cc1Nc1ccccc1</smiles>

8a

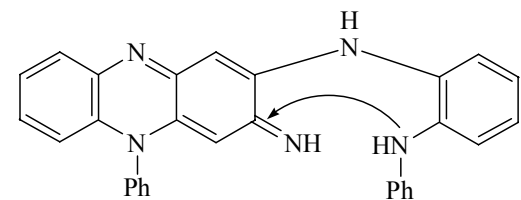

14<smiles>NC12C=C3C(=CC1=Nc1ccccc1N3c1ccccc1)Nc1ccccc1N2c1ccccc1</smiles>

$[\mathrm{O}]$<smiles>N=C1C=C(N)/C(=N\c2ccccc2Nc2ccccc2)C=C1Nc1ccccc1-c1ccccc1</smiles>

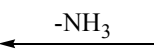

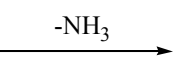<smiles></smiles>

3a

Scheme 3

\section{Conclusions}

We have developed a regiospecific and high yielding synthesis for 5,7-disubstituted quinoxalino $[2,3-b]$ phenazines. The synthetic route makes these unusual zwitterions readily available for further study.

\section{Experimental Section}

General Procedures. Reactions and column eluents were monitored by TLC using plasticbacked thin layer chromatography plates (Kodak) viewed under UV light at 254 and $350 \mathrm{~nm}$. Dry flash chromatography on Bodman flash silica 32-63 was used for separations. UV/vis spectra were measured on HP 8453 UV-visible spectrometer. IR spectra were measured on a Mattson Infinity Series FTIR spectrometer. ${ }^{1} \mathrm{H}$ and ${ }^{13} \mathrm{C}$ NMR spectra were measured on Brucker AMX500, AMX400 and AC200 machines. Mass spectra were recorded on VG ZAB-SE or Autospec “Q” machines. Microanalyses were carried out by Desert Analytics, Inc. 


\section{Preparation of $\mathrm{N}$-aryl substituted 2-nitrobenzamines}

$N$-(4-n-Butylphenyl)-2-nitrobenzamine. To a stirred mixture of 1-fluoro-2-nitrobenzene $(3.33 \mathrm{~mL}, 31.60 \mathrm{mmol})$ and $4-n$-butylaniline $(10 \mathrm{~mL}, 63.32 \mathrm{mmol})$ at $c a .20 \mathrm{C}$, under argon, potassium fluoride $(1.9 \mathrm{~g}, 32.76 \mathrm{mmol})$ was added in one portion. The reaction mixture was heated at $c a .180 \mathrm{C}$ for $48 \mathrm{~h}$ and then allowed to cool to $c a .20 \mathrm{C}$. The mixture was dissolved in dichloromethane and extracted with dilute aqueous hydrochloric acid (5-10\%) to remove unreacted amine. The organic layer was separated, dried $\left(\mathrm{MgSO}_{4}\right)$, and filtered through fluted filter paper. Dry flash chromatography gave the title compound $(7 \mathrm{~g}, 82 \%)$ as a red oil (Found: $\mathrm{C}$, 71.38; $\mathrm{H}, 6.51 ; \mathrm{N}, 10.35 . \mathrm{C}_{16} \mathrm{H}_{18} \mathrm{~N}_{2} \mathrm{O}_{2}$ requires $\left.\mathrm{C}, 71.11 ; \mathrm{H}, 6.67 ; \mathrm{N}, 10.37 \%\right) ; \lambda_{\max }(\mathrm{DCM}) / \mathrm{nm}$ 230 (log $\varepsilon$ 4.10), 260 (4.15), $285 \inf$ (4.08), 437 (3.83); $v_{\max }(\mathrm{Drift}) / \mathrm{cm}^{-1} 3369 \mathrm{~m}$ and 3359m (Ar $\mathrm{NH}), 3078 \mathrm{~m}, 3039 \mathrm{~m}$ and $3028 \mathrm{~m}(\mathrm{Ar} \mathrm{CH}), 2964 \mathrm{~s}, 2943 \mathrm{~s}, 2887 \mathrm{~s}$ and $2863 \mathrm{~s}\left(\mathrm{CH}_{2}\right.$ and $\left.\mathrm{CH}_{3}\right)$, $1626 \mathrm{~s}, 1579 \mathrm{~s}, 1522 \mathrm{~s}, 1502 \mathrm{~m}, 1446 \mathrm{~m}, 1408 \mathrm{~m}, 1356 \mathrm{~s}, 1331 \mathrm{~m}, 1279 \mathrm{~s}, 1234 \mathrm{~m}, 1165 \mathrm{~m}, 1153 \mathrm{~m}$, $1119 \mathrm{~m}, 1080 \mathrm{~m}, 1043 \mathrm{~m}, 1020 \mathrm{~m}, 953 \mathrm{w}, 930 \mathrm{w}, 893 \mathrm{~m}, 849 \mathrm{~s}, 781 \mathrm{~s}, 748 \mathrm{~s}, 696 \mathrm{~m}, 628 \mathrm{~m}$; $\delta_{\mathrm{H}}\left(200 \mathrm{MHz} ; \mathrm{CD}_{2} \mathrm{Cl}_{4}\right) 9.45(1 \mathrm{H}$, br s, NH), $8.18(1 \mathrm{H}, \mathrm{d}, J 8.6 \mathrm{~Hz}, \operatorname{Ar} \mathbf{H}), 7.36(1 \mathrm{H}, \mathrm{dd}, J$ 7.7, 7.6 Hz, Ar H), 7.28-7.16 (5H, m, Ar H), 6.75 (1H, dd, J 7.9, 7.8 Hz, Ar H), 2.66 (2H, t, J 7.4 Hz, $\left.\mathrm{ArCH}_{2}\right), 1.72-1.57\left(2 \mathrm{H}, \mathrm{m}, \mathrm{CH}_{2}\right), 1.49-1.30\left(2 \mathrm{H}, \mathrm{m}, \mathrm{CH}_{2}\right), 0.97(3 \mathrm{H}, \mathrm{t}, J 7.2 \mathrm{~Hz}, \mathrm{CH})$; $\delta_{\mathrm{C}}\left(50 \mathrm{MHz} ; \mathrm{CD}_{2} \mathrm{Cl}_{2}\right)$ 143.97, 141.17, 136.59, 135.99 (Ar CH), 133.34, 129.98 (Ar CH), 126.81 (Ar CH), 124.97 (Ar CH), 117.45 (Ar CH), 116.39 (Ar CH), $35.69\left(\mathrm{ArCH}_{2}\right), 34.26\left(\mathbf{C H}_{2}\right), 22.95$ $\left(\mathrm{CH}_{2}\right), 14.33\left(\mathrm{CH}_{3}\right) ; \mathrm{m} / z(\mathrm{FAB}) 270\left(\mathrm{M}^{+}, 100 \%\right)$ (Found: $\mathrm{M}^{+}, 270.1366 . \mathrm{C}_{16} \mathrm{H}_{18} \mathrm{~N}_{2} \mathrm{O}_{2}$ requires $M$, 270.1368).

$\mathbf{N}$-(4-t-Butylphenyl)-2-nitrobenzamine. Similarly the treatment of 1-fluoro-2-nitrobenzene with 4-t-butylaniline gave the title compound (95\%) as a red oil (Found: C, 71.34; H, 6.59; N, 10.29. $\mathrm{C}_{16} \mathrm{H}_{18} \mathrm{~N}_{2} \mathrm{O}_{2}$ requires $\left.\mathrm{C}, 71.11 ; \mathrm{H}, 6.67 ; \mathrm{N}, 10.37 \%\right) ; \lambda_{\max }(\mathrm{DCM}) / \mathrm{nm} 232$ (log $\varepsilon$ 4.09), 259 (4.16), 285 inf (4.03), 439 (3.82); $v_{\max }(\mathrm{Drift}) / \mathrm{cm}^{-1} 3367 \mathrm{~m}, 3357 \mathrm{~m}$ and $3342 \mathrm{~m}(\mathrm{Ar} \mathrm{NH}), 3078 \mathrm{~m}$ and $3041 \mathrm{~m}(\mathrm{Ar} \mathrm{CH}), 2978 \mathrm{~s}, 2943 \mathrm{~s}, 2902 \mathrm{~s}$ and 2873s $\left(\mathrm{CH}_{3}\right), 1624 \mathrm{~s}, 1583 \mathrm{~m}, 1523 \mathrm{~s}, 1504 \mathrm{w}, 1446 \mathrm{~m}$, $1431 \mathrm{~m}, 1367 \mathrm{~m}, 1356 \mathrm{~m}, 1331 \mathrm{~m}, 1281 \mathrm{~s}, 1236 \mathrm{w}, 1167 \mathrm{~m}, 1153 \mathrm{~m}, 1117 \mathrm{~m}, 1080 \mathrm{~m}, 1045 \mathrm{~m}, 950 \mathrm{w}$, $895 \mathrm{~m}, 858 \mathrm{~m}, 810 \mathrm{~m}, 781 \mathrm{~m}, 748 \mathrm{~s}, 690 \mathrm{~m}, 617 \mathrm{~m} ; \delta_{\mathrm{H}}\left(200 \mathrm{MHz} ; \mathrm{CD}_{2} \mathrm{Cl}_{2}\right) 9.45(1 \mathrm{H}$, br s, NH), 8.18 $(1 \mathrm{H}, \mathrm{d}, J 8.7 \mathrm{~Hz}, \operatorname{Ar} \mathbf{H}), 7.46(2 \mathrm{H}, \mathrm{d}, J 6.6 \mathrm{~Hz}, \operatorname{Ar} \mathbf{H}), 7.37(1 \mathrm{H}, \mathrm{dd}, J 7.8,7.8 \mathrm{~Hz}, \operatorname{Ar} \mathbf{H}), 7.25-$ $7.19(3 \mathrm{H}, \mathrm{m}, \mathrm{Ar} \mathbf{H}), 6.76(1 \mathrm{H}, \mathrm{dd}, J 7.7,7.7 \mathrm{~Hz}, \mathrm{Ar} \mathbf{H}), 1.36\left(9 \mathrm{H}, \mathrm{s}, \mathrm{CH}_{3}\right) ; \delta_{\mathrm{C}}\left(50 \mathrm{MHz} ; \mathrm{CD}_{2} \mathrm{Cl}_{2}\right)$ 149.28, 143.88, 136.40, 136.00 (Ar CH), 133.61, 126.94 (Ar CH), 126.81 (Ar CH), $124.53(\mathrm{Ar}$ CH), 117.51 (Ar CH), 116.44 (Ar CH), $34.82\left(\mathbf{C m e}_{3}\right), 31.50\left(\mathbf{C H}_{3}\right) ; m / z(\mathrm{FAB}) 270\left(\mathrm{M}^{+}, 100 \%\right)$ (Found: $\mathrm{M}^{+}, 270.1362 . \mathrm{C}_{16} \mathrm{H}_{18} \mathrm{~N}_{2} \mathrm{O}_{2}$ requires $M, 270.1368$ ).

\section{Preparation of $N$-alkyl substituted 2-nitrobenzamines}

$\boldsymbol{N}$-(n-Butyl)-2-nitrobenzamine. To a stirred mixture of 1-fluoro-2-nitrobenzene (2.68g, $18.98 \mathrm{mmol})$ and $n$-butylamine $(11.8 \mathrm{~mL}, 12 \mathrm{mmol})$ in EtOH $(30 \mathrm{~mL})$ at ca. $20 \mathrm{C}$, under argon, Hünig's base $(2.1 \mathrm{~mL}, 12 \mathrm{mmol})$ was added in one portion. The reaction mixture was heated to reflux $(c a .80 \mathrm{C}$ ) for $24 \mathrm{~h}$ and then allowed to cool to $c a .20 \mathrm{C}$. The mixture was diluted with $\mathrm{DCM}$ and extracted with dilute aqueous $\mathrm{HCl}(5-10 \%)$ to remove unreacted amines. The organic layer was separated, dried $\left(\mathrm{MgSO}_{4}\right)$ and filtered. Dry flash chromatography gave the title 
compound $\left(2.68 \mathrm{~g}, 87 \%\right.$ ) as an orange oil (Found: $\mathrm{C}, 62.07 ; \mathrm{H}, 7.27 ; \mathrm{N}, 14.16 . \mathrm{C}_{10} \mathrm{H}_{14} \mathrm{~N}_{2} \mathrm{O}_{2}$ requires $\mathrm{C}, 61.86 ; \mathrm{H}, 7.22 ; \mathrm{N}, 14.43 \%) ; \lambda_{\max }(\mathrm{DCM}) / \mathrm{nm} 242$ (log $\varepsilon$ 4.02), 280 (3.60), 435 (3.60); $v_{\max }(\mathrm{Drift}) / \mathrm{cm}^{-1} 3392 \mathrm{~m}$ (Ar NH), 3086w and 3057w (Ar CH), 2970m, 2939m and $2877 \mathrm{~m}\left(\mathrm{CH}_{2}\right.$ and $\left.\mathrm{CH}_{3}\right), 1627 \mathrm{~s}, 1581 \mathrm{~s}, 1539 \mathrm{~s}, 1522 \mathrm{~m}, 1479 \mathrm{~m}, 1446 \mathrm{~m}, 1425 \mathrm{~m}, 1363 \mathrm{~s}, 1282 \mathrm{~s}, 1245 \mathrm{w}, 1203 \mathrm{w}$, $1171 \mathrm{~m}, 1117 \mathrm{w}, 1076 \mathrm{w}, 1043 \mathrm{w}, 953 \mathrm{w}, 866 \mathrm{~s}, 810 \mathrm{~m}, 783 \mathrm{~s}, 752 \mathrm{~s}, 730 \mathrm{w}, 696 \mathrm{~m}, 659 \mathrm{~m}$; $\delta_{\mathrm{H}}\left(200 \mathrm{MHz} ; \mathrm{CD}_{2} \mathrm{Cl}_{2}\right) 8.10(1 \mathrm{H}, \mathrm{d}, J 8.6 \mathrm{~Hz}, \mathrm{Ar} \mathrm{H}), 8.04(1 \mathrm{H}, \mathrm{br} \mathrm{s}, \mathrm{NH}), 7.43(1 \mathrm{H}, \mathrm{dd}, J$ 7.7, $7.5 \mathrm{~Hz}, \operatorname{Ar} \mathbf{H}), 6.86(1 \mathrm{H}, \mathrm{d}, J 6.7 \mathrm{~Hz}, \operatorname{Ar} \mathbf{H}), 6.61(1 \mathrm{H}, \mathrm{dd}, J$ 7.7, $7.6 \mathrm{~Hz}, \operatorname{Ar} \mathbf{H}), 3.29(2 \mathrm{H}$, quartet, $\left.\mathrm{NCH}_{2}\right), 1.70\left(2 \mathrm{H}\right.$, quintet, $\left.\mathbf{C H}_{2}\right), 1.47\left(2 \mathrm{H}\right.$, hextet, $\left.\mathrm{CH}_{2}\right), 0.98\left(3 \mathrm{H}, \mathrm{t}, J 7.2 \mathrm{~Hz}, \mathrm{CH}_{3}\right)$; $\delta_{\mathrm{C}}\left(50 \mathrm{MHz} ; \mathrm{CD}_{2} \mathrm{Cl}_{2}\right) 146.05$ (Ar CNHR), $136.48(\mathrm{Ar} \mathrm{CH}), 132.07\left(\mathrm{Ar} \mathbf{C N O}_{2}\right), 126.94(\mathrm{Ar} \mathrm{CH})$, $115.26(\mathrm{Ar} \mathbf{C H}), 114.27(\mathrm{Ar} \mathbf{C H}), 43.10\left(\mathrm{NCH}_{2}\right), 31.41\left(\mathrm{CH}_{2}\right), 20.61\left(\mathrm{CH}_{2}\right), 13.91\left(\mathrm{CH}_{3}\right) ; \mathrm{m} / \mathrm{z}$ (EI) $194\left(\mathrm{M}^{+}, 69 \%\right)$ (Found: $\mathrm{M}^{+}, 194.1055 . \mathrm{C}_{10} \mathrm{H}_{14} \mathrm{~N}_{2} \mathrm{O}_{2}$ requires $\left.M, 194.1055\right)$.

\section{Preparation of $N$-substituted 1,2-benzenediamines}

$\mathrm{N}$-(4-n-Butylphenyl)-1,2-benzenediamine (5b). To a stirred solution of $\mathrm{N}$-(4-n-butylphenyl)-2nitrobenzamine $(1.96 \mathrm{~g}, 7.26 \mathrm{mmol})$ in EtOH $(50 \mathrm{ml})$ at $c a .20 \mathrm{C}$, under argon, $(10 \%)$ palladium on carbon $(500 \mathrm{mg}$ ) was added in one portion. The reaction mixture was evacuated (to $25 \mathrm{mmHg}$ ) and flushed with argon 3 times then the mixture was evacuated (to $25 \mathrm{mmHg}$ ) and flushed with hydrogen 3 times. The reaction mixture was then left to stir under an atmosphere of hydrogen. The color of the mixture became dark red and after $1 \mathrm{~h}$ this red color disappeared and consumption of hydrogen had ceased. The mixture was filtered through a celite pad to remove palladium residues and the filtrate was diluted with water until a flocculant cream colored percipitate was obtained. Filtration gave the title compound $5 \mathbf{b}(1.69 \mathrm{~g}, 97 \%)$ as cream colored solid, mp 72-73.5 C (from EtOH/water) (Found: C, 80.14; H, 8.51; N, 11.74. $\mathrm{C}_{16} \mathrm{H}_{20} \mathrm{~N}_{2}$ requires $\mathrm{C}, 80.00 ; \mathrm{H}, 8.33 ; \mathrm{N}, 11.67 \%) ; \lambda_{\max }(\mathrm{DCM}) / \mathrm{nm} 237$ (log $\varepsilon$ 4.20), 272 (3.91), 296 (3.87); $v_{\max }(\mathrm{Drift}) / \mathrm{cm}^{-1} 3425 \mathrm{~m}, 3340 \mathrm{~s}$ and 3313s (Ar NH), 3033w (Ar CH), 2953s, 2924s, 2868m, 2852s $\left(\mathrm{CH}_{2}\right.$ and $\left.\mathrm{CH}_{3}\right), 1616 \mathrm{~s}, 1558 \mathrm{w}, 1520 \mathrm{~s}, 1466 \mathrm{~m}, 1456 \mathrm{~m}, 1444 \mathrm{~m}, 1402 \mathrm{~m}, 1375 \mathrm{w}, 1317 \mathrm{~s}, 1300 \mathrm{~m}$, $1259 \mathrm{~m}, 1246 \mathrm{w}, 1246 \mathrm{w}, 1221 \mathrm{w}, 1203 \mathrm{w}, 1178 \mathrm{w}, 1134 \mathrm{w}, 1120 \mathrm{~m}, 1059 \mathrm{w}, 928 \mathrm{w}, 887 \mathrm{w}, 863 \mathrm{w}$, $825 \mathrm{~m}, 750 \mathrm{~m}, 649 \mathrm{w} ; \delta_{\mathrm{H}}\left(200 \mathrm{MHz} ; \mathrm{CD}_{2} \mathrm{Cl}_{2}\right)$ 7.13-6.96 (4H, m, $\left.\mathrm{Ar} \mathbf{H}\right), 6.83-6.67$ (4H, m, $\left.\mathrm{Ar} \mathbf{H}\right)$, $5.22(1 \mathrm{H}$, br s, $\mathrm{NH}), 3.80\left(2 \mathrm{H}\right.$, br s, $\left.\mathrm{NH}_{2}\right), 2.56\left(2 \mathrm{H}, \mathrm{t}, J 7.6 \mathrm{~Hz}, \mathrm{NCH}_{2}\right), 1.59$ (2H, quintet, $\left.\mathrm{CH}_{2}\right)$, 1.39 ( $2 \mathrm{H}$, heptet, $\left.\mathrm{CH}_{2}\right), 0.97\left(3 \mathrm{H}, \mathrm{t}, J 7.1 \mathrm{~Hz}, \mathrm{CH}_{3}\right) ; \delta_{\mathrm{C}}\left(50 \mathrm{MHz} ; \mathrm{CD}_{2} \mathrm{Cl}_{2}\right) 143.51,142.07,134.43$, 129.84, 129.48 (Ar CH), 125.41 (Ar CH), 124.23 (Ar CH), 119.29 (Ar CH), 116.38 (Ar CH), 116.01 ( $\mathrm{Ar} \mathrm{CH}), 35.14\left(\mathrm{NCH}_{2}\right), 34.41\left(\mathrm{CH}_{3}\right), 22.76\left(\mathrm{CH}_{2}\right), 14.15\left(\mathrm{CH}_{3}\right) ; m / z(\mathrm{EI}) 240\left(\mathrm{M}^{+}\right.$, $100 \%$ ) (Found: $\mathrm{M}^{+}, 240.1628 . \mathrm{C}_{16} \mathrm{H}_{20} \mathrm{~N}_{2}$ requires $M, 240.1626$ ).

$N$-(4-t-Butylphenyl)-1,2-benzenediamine (5c). Similarly hydrogenation of $N$-(4-t-butylphenyl)2-nitrobenzamine gave the title compound 5c (95\%) as cream colored solid, mp 82-85 C (from EtOH/water) (Found: C, 79.95; H, 8.19; N, 11.79. $\mathrm{C}_{16} \mathrm{H}_{20} \mathrm{~N}_{2}$ requires $\mathrm{C}, 80.00 ; \mathrm{H}, 8.33 ; \mathrm{N}$, 11.67\%); $\lambda_{\max }(\mathrm{DCM}) / \mathrm{nm} 237$ (log $\left.\varepsilon 4.15\right), 273$ (3.83), 296 (3.80); $v_{\max }(\mathrm{Drift}) / \mathrm{cm}^{-1} 3425 \mathrm{~m}$ and $3342 \mathrm{~s}(\mathrm{Ar} \mathrm{NH}), 3043 \mathrm{w}$ and $3022 \mathrm{~m}(\mathrm{Ar} \mathrm{CH}), 2962 \mathrm{~s}, 2904 \mathrm{~m}$ and $2866 \mathrm{~m}\left(\mathrm{CH}_{2}\right.$ and $\left.\mathrm{CH}_{3}\right), 1612 \mathrm{~s}$, $1591 \mathrm{~m}, 1515 \mathrm{~s}, 1500 \mathrm{~s}, 1464 \mathrm{~m}, 1442 \mathrm{~m}, 1394 \mathrm{w}, 1363 \mathrm{w}, 1304 \mathrm{~s}, 1257 \mathrm{~m}, 1221 \mathrm{w}, 1192 \mathrm{w}, 1136 \mathrm{w}$, $1124 \mathrm{w}, 1059 \mathrm{w}, 1010 \mathrm{w}, 929 \mathrm{w}, 887 \mathrm{w}, 865 \mathrm{w}, 825 \mathrm{~m}, 750 \mathrm{~m}, 692 \mathrm{w}, 644 \mathrm{w} ; \delta_{\mathrm{H}}\left(200 \mathrm{MHz} ; \mathrm{CD}_{2} \mathrm{Cl}_{2}\right)$ 
$7.28(2 \mathrm{H}, \mathrm{d}, J 6.8 \mathrm{~Hz}, \operatorname{Ar} \mathbf{H}), 7.13(1 \mathrm{H}, \mathrm{d}, J 7.8 \mathrm{~Hz}, \operatorname{Ar} \mathbf{H}), 7.02(1 \mathrm{H}, \mathrm{dd}, J 7.7,7.6 \mathrm{~Hz}, \operatorname{Ar} \mathbf{H})$, $6.82(1 \mathrm{H}, \mathrm{d}, J 6.7 \mathrm{~Hz}, \mathrm{Ar} \mathbf{H}), 6.73(3 \mathrm{H}, \mathrm{m}, \operatorname{Ar} \mathbf{H}), 5.25(1 \mathrm{H}, \mathrm{br} \mathrm{s}, \mathrm{NH}), 3.80\left(2 \mathrm{H}\right.$, br s, $\left.\mathrm{NH}_{2}\right), 1.34$ $\left(9 \mathrm{H}, \mathrm{s}, \mathrm{CH}_{3}\right) ; \delta_{\mathrm{C}}\left(50 \mathrm{MHz} ; \mathrm{CD}_{2} \mathrm{Cl}_{2}\right) 143.33,142.65,142.21,129.72,126.43(\mathrm{Ar} \mathrm{CH}), 125.55(\mathrm{Ar}$ CH), 124.44 ( $\mathrm{Ar} \mathrm{CH}), 119.30(\mathrm{Ar} \mathrm{CH}), 116.40(\mathrm{Ar} \mathbf{C H}), 115.62(\mathrm{Ar} \mathbf{C H}), 34.31\left(\mathrm{Cme}_{3}\right), 31.72$ $\left(\mathrm{CH}_{3}\right) ; m / z$ (EI) $240\left(\mathrm{M}^{+}, 100 \%\right)$ (Found: $\mathrm{M}^{+}, 240.1622$. $\mathrm{C}_{16} \mathrm{H}_{20} \mathrm{~N}_{2}$ requires $\left.M, 240.1626\right)$.

$\mathrm{N}$-(n-Butyl)-1,2-benzenediamine (5d). Similarly hydrogenation of $\mathrm{N}$-( $n$-butyl)-2nitrobenzamine gave the title compound $\mathbf{5 d}(97 \%)$ as a dark colored oil that solidifies on standing, mp 33-35 C (crude) (Found: C, 73.24; H, 9.52; N, 17.14. $\mathrm{C}_{10} \mathrm{H}_{16} \mathrm{~N}_{2}$ requires $\mathrm{C}, 73.17$; H, 9.76; N, 17.07\%); $\lambda_{\max }(\mathrm{DCM}) / \mathrm{nm} 229$ (log $\left.\varepsilon 3.92\right), 249$ (3.91), 298 (3.57); $v_{\max }(\mathrm{Drift}) / \mathrm{cm}^{-1}$ $3388 \mathrm{~s}, 3356 \mathrm{~s}, 3317 \mathrm{~s}, 3265 \mathrm{~m}$ and $3234 \mathrm{~m}(\mathrm{Ar} \mathrm{NH}), 3066 \mathrm{~m}, 3045 \mathrm{~m}$ and $3032 \mathrm{~m}(\mathrm{Ar} \mathrm{CH}), 2966 \mathrm{~s}$, 2941s, and 2873s $\left(\mathrm{CH}_{2}\right.$ and $\left.\mathrm{CH}_{3}\right), 1635 \mathrm{~m}, 1606 \mathrm{~m}, 1575 \mathrm{~m}, 1558 \mathrm{~m}, 1522 \mathrm{~m}, 1472 \mathrm{~m}, 1458 \mathrm{~m}$, $1377 \mathrm{~m}, 1363 \mathrm{~m}, 1340 \mathrm{~m}, 1315 \mathrm{~m}, 1279 \mathrm{~m}, 1230 \mathrm{~m}, 1207 \mathrm{~m}, 1151 \mathrm{~m}, 1115 \mathrm{~m}, 1043 \mathrm{~m}, 908 \mathrm{~m}, 848 \mathrm{~m}$, $754 \mathrm{~s}, 687 \mathrm{~m}, 642 \mathrm{~m} ; \delta_{\mathrm{H}}\left(200 \mathrm{MHz} ; \mathrm{CD}_{2} \mathrm{Cl}_{2}\right) 6.87-6.63(4 \mathrm{H}, \mathrm{m}, \mathrm{Ar} \mathbf{H}), 3.36(3 \mathrm{H}$, br s, NH and $\left.\mathrm{NH}_{2}\right), 3.14\left(2 \mathrm{H}, \mathrm{t}, J 7.0 \mathrm{~Hz}, \mathrm{NCH}_{2}\right), 1.69\left(2 \mathrm{H}\right.$, quintet, $\left.\mathrm{CH}_{2}\right), 1.52\left(2 \mathrm{H}\right.$, hextet, $\left.\mathrm{CH}_{2}\right), 1.03(3 \mathrm{H}, \mathrm{t}$, $\left.J 7.2 \mathrm{~Hz}, \mathrm{CH}_{3}\right) ; \delta_{\mathrm{C}}\left(50 \mathrm{MHz} ; \mathrm{CD}_{2} \mathrm{Cl}_{2}\right)$ 138.73, 134.90, $121.02(\mathrm{Ar} \mathrm{CH}), 118.70(\mathrm{Ar} \mathrm{CH}), 116.71$ (Ar CH), $112.00(\mathrm{Ar} \mathbf{C H}), 44.58\left(\mathrm{NCH}_{2}\right), 32.45\left(\mathrm{CH}_{2}\right), 21.08\left(\mathrm{CH}_{2}\right), 14.40\left(\mathrm{CH}_{3}\right) ; \mathrm{m} / z(\mathrm{EI}) 164$ $\left(\mathrm{M}^{+}, 100 \%\right.$ ) (Found: $\mathrm{M}^{+}, 164.1312 . \mathrm{C}_{10} \mathrm{H}_{16} \mathrm{~N}_{2}$ requires $\left.M, 164.1313\right)$.

\section{Preparation of 5-fluoro-2,4-dinitrobenzamines}

1-[N-( $N$ '-phenyl-1,2-benzenediamino)]-5-fluoro-2,4-dinitrobenzene (9). To a stirred solution of 1,5-difluoro-2,4-dinitrobenzene $6(6.63 \mathrm{~g}, 32.5 \mathrm{mmol})$ in $\mathrm{EtOH}(200 \mathrm{~mL})$ at $c a .0 \mathrm{C}$, under argon, $N$-phenyl-1,2-benzenediamine $5 \mathrm{a}(5.98 \mathrm{~g}, 32.5 \mathrm{mmol})$ was added in several portions. The color of the reaction mixture became orange and within $2 \mathrm{~h}$ an orange crystalline precipitate was observed. To the cooled reaction mixture ( $c a .0 \mathrm{C}$ ) Hünig's base $(5.65 \mathrm{~mL}, 32.44 \mathrm{mmol})$ was added in three portions over a period of $2 \mathrm{~h}$ and on complete addition the mixture was left to warm to $c a .20 \mathrm{C}$. The orange precipitate was removed by filtration, washed $\left(\mathrm{H}_{2} \mathrm{O}\right)$ and dried to afford the title compound $9(11.42 \mathrm{~g}, 95 \%$ ) as orange red needles, mp 164-166 C (from EtOH) (Found: $\mathrm{C}, 58.52 ; \mathrm{H}, 3.56 ; \mathrm{N}, 14.63 . \mathrm{C}_{18} \mathrm{H}_{13} \mathrm{FN}_{4} \mathrm{O}_{4} .1 / 2 \mathrm{C}_{2} \mathrm{H}_{6} \mathrm{O}$ requires $\mathrm{C}, 58.31 ; \mathrm{H}, 4.09 ; \mathrm{N}$, 14.32\%); $\lambda_{\max }(\mathrm{DCM}) / \mathrm{nm} 230$ (log $\left.\varepsilon 4.28\right), 276$ (4.39), 327 (4.18), $360 \mathrm{inf}(4.03) ; v_{\max }(\mathrm{Drift}) / \mathrm{cm}^{-1}$ 3379s and 3300s (Ar NH), 3101w and 3039w (Ar CH), 1643m, 1620s, 1597s, 1545s, 1518s, $1500 \mathrm{~s}, 1477 \mathrm{~s}, 1464 \mathrm{~s}, 1421 \mathrm{~s}, 1367 \mathrm{~s}, 1336 \mathrm{~s}, 1327 \mathrm{~s}, 1271 \mathrm{~s}, 1236 \mathrm{~m}, 1221 \mathrm{~m}, 1132 \mathrm{~m}, 1051 \mathrm{~m}, 928 \mathrm{w}$, $897 \mathrm{w}, 885 \mathrm{w}, 860 \mathrm{w}, 831 \mathrm{w}, 752 \mathrm{~m}, 719 \mathrm{w}, 700 \mathrm{~m}, 677 \mathrm{w}, 642 \mathrm{w}, 604 \mathrm{~m} ; \delta_{\mathrm{H}}\left(200 \mathrm{MHz} ; \mathrm{DMSO}-d_{6}\right)$ $10.02\left(1 \mathrm{H}\right.$, br s, NH), $8.94\left(1 \mathrm{H}, \mathrm{d}, J_{\mathrm{HF}} 8.0 \mathrm{~Hz}, \mathrm{Ar} \mathbf{H}-3\right), 7.83(1 \mathrm{H}, \mathrm{br} \mathrm{s}, \mathrm{NH}), 7.38-7.19(5 \mathrm{H}, \mathrm{m}$, $\operatorname{Ar} \mathbf{H}), 7.08-6.97(3 \mathrm{H}, \mathrm{m}, \mathrm{Ar} \mathbf{H}), 6.88(1 \mathrm{H}, \mathrm{dd}, J 7.2,7.1 \mathrm{~Hz}, \operatorname{Ar} \mathbf{H}), 6.55\left(1 \mathrm{H}, \mathrm{d}, J_{\mathrm{HF}} 14.2 \mathrm{~Hz}, \mathrm{Ar}\right.$ H-6); observable peaks $\delta_{\mathrm{C}}\left(50 \mathrm{MHz}\right.$; DMSO- $\left.d_{6}\right) 158.60$ (d, $\left.J_{\mathrm{CF}} 264.9 \mathrm{~Hz}, \mathrm{Ar} \mathrm{CF}\right), 148.39$ (d, $J_{\mathrm{CF}}$ $13.3 \mathrm{~Hz}, \mathrm{Ar} \mathbf{C N H}), 142.52$ (Ar CNH), 139.87 (Ar CNH), 129.01 (Ar CH), 128.76 (Ar CH), 128.45 (Ar CH), 128.26, 126.85 (Ar CH), 125.80, 125.67, 125.61, 120.90 (d, $J_{\mathrm{CF}} 12.3 \mathrm{~Hz}, \mathrm{Ar}$ CH-6), 118.72 (Ar CH), 117.92 (Ar CH), 103.06 (d, $\left.J_{\mathrm{CF}} 27.1 \mathrm{~Hz}, \mathrm{Ar} \mathrm{CH}-3\right) ; m / z(\mathrm{FAB}) 368\left(\mathrm{M}^{+}\right.$, 100\%) (Found: $\mathrm{M}^{+}, 368.0922 . \mathrm{C}_{18} \mathrm{H}_{13} \mathrm{FN}_{4} \mathrm{O}_{4}$ requires $M$, 368.0921). 


\section{Preparation of 1,5-bisamino-2,4-dinitrobenzenes}

1,5-Bis[ $N$-( $N$ '-phenyl-1,2-benzenediamino)]-2,4-dinitrobenzene (7a). Method 1. To a stirred solution of $N$-phenyl-1,2-benzenediamine 5a $(14.75 \mathrm{~g}, 80 \mathrm{mmol})$ in EtOH $(120 \mathrm{~mL})$ at ca. $20 \mathrm{C}$, under argon, 1,5-difluoro-2,4-dinitrobenzene 6 (4.08g, 20mmol) was added in one portion. The color of the reaction mixture became red and within $15 \mathrm{~min}$ a red crystalline precipitate was observed. The reaction mixture was heated under reflux for $12 \mathrm{~h}$, then allowed to cool to $c a$. $20 \mathrm{C}$. The red precipitate was filtered, washed with hot water and then with cold EtOH and dried to afford compound 7a $(10.48 \mathrm{~g}, 98.5 \%)$ as brick red prisms, mp 219-220 C (from EtOH) (Found: C, 67.88; H, 4.47; N, 15.94. $\mathrm{C}_{30} \mathrm{H}_{24} \mathrm{~N}_{6} \mathrm{O}_{4}$ requires $\left.\mathrm{C}, 67.67 ; \mathrm{H}, 4.51 ; \mathrm{N}, 15.79 \%\right) ; \lambda_{\max }(\mathrm{DCM}) / \mathrm{nm}$ 231 ( $\log \varepsilon$ 4.46), 283 (4.59), 331 (4.45), $369 \mathrm{inf}$ (4.29); $v_{\max }(\mathrm{Drift}) / \mathrm{cm}^{-1} 3402 \mathrm{~s}, 3379 \mathrm{~s}, 3336 \mathrm{~s}$ and 3321s (Ar NH), 3186w, 3089m, 3077m and 3046m (Ar CH), 1605m, 1592m, 1585m, 1564m, $1555 \mathrm{~m}, 1530 \mathrm{~m}, 1513 \mathrm{~m}, 1493 \mathrm{~m}, 1484 \mathrm{~m}, 1463 \mathrm{~m}, 1424 \mathrm{~m}, 1335 \mathrm{~m}, 1318 \mathrm{~m}, 1279 \mathrm{~m}, 1254 \mathrm{~m}, 1161 \mathrm{~m}$, $1104 \mathrm{~m}, 1069 \mathrm{~m}, 1028 \mathrm{w}, 971 \mathrm{w}, 897 \mathrm{w}, 744 \mathrm{~m}, 690 \mathrm{~m} ; \delta_{\mathrm{H}}\left(400 \mathrm{MHz} ; \mathrm{DMSO}-d_{6}\right) 9.42(2 \mathrm{H}, \mathrm{s}, \mathrm{NH})$, 8.98 (1H, s, H-3), 7.61 (2H, s, PhNH), 7.17-7.10 (10H, m, Ar H), 6.89-6.78 (8H, m, Ar H), 5.97 $(1 \mathrm{H}, \mathrm{s}, \mathbf{H}-6)$; one carbon signal missing $\delta_{\mathrm{C}}\left(100 \mathrm{MHz}\right.$; DMSO- $\left.d_{6}\right) 146.60(\mathrm{Ar} \mathrm{CNH}), 142.80(\mathrm{Ar}$ CNH), 139.28 (Ar CNH), 128.92, 127.57, 127.52, 127.38, 126.65, 125.03, 120.56, 118.28, 117.69, 95.01 (CH-3); m/z (FAB) $532\left(\mathrm{M}^{+}, 100 \%\right.$ ) (Found: $\mathrm{M}^{+}, 532.1876 . \mathrm{C}_{30} \mathrm{H}_{24} \mathrm{~N}_{6} \mathrm{O}_{4}$ requires $M, 532.1859)$.

Method 2. To a stirred solution of $N$-phenyl-1,2-benzenediamine 5a (7.38g, 40mmol) in EtOH $(120 \mathrm{~mL})$ at $c a .20 \mathrm{C}$, under argon, 1,5-difluoro-2,4-dinitrobenzene 6 (4.08g, 20mmol) was added in one portion. The color of the reaction mixture became orange-red and Hünig's base $(7 \mathrm{~mL}$, $40 \mathrm{mmol}$ ) was then added in one portion. Within $30 \mathrm{~min}$. a red crystalline precipitate was observed. The reaction mixture was heated under reflux for $24 \mathrm{~h}$, then allowed to cool to $c a$. $20 \mathrm{C}$. The red precipitate was filtered, washed with hot water and then with cold $\mathrm{EtOH}$ and dried to afford compound 7a $(9.68 \mathrm{~g}, 91 \%$ ) as brick red prisms, mp 219-220 C (from EtOH), identical to an authentic sample.

1,5-Bis[ $N$-( $N^{\prime}$-4-n-butylphenyl-1,2-benzenediamino)]-2,4-dinitrobenzene (7b). Similarly (Method 2) the treatment of 1,5-difluoro-2,4-dinitrobenzene 6 with $N$-(4-n-butylphenyl)-1,2benzenediamine $5 \mathbf{b}$ and Hünig's base gave the title compound $\mathbf{7 b}(85 \%)$ as a brick red precipitate, mp 166.5-169 C (from EtOH) (Found: $\mathrm{C}, 71.11 ; \mathrm{H}, 6.17 ; \mathrm{N}, 13.34 . \mathrm{C}_{38} \mathrm{H}_{40} \mathrm{~N}_{6} \mathrm{O}_{4}$ requires $\mathrm{C}, 70.81 ; \mathrm{H}, 6.21 ; \mathrm{N}, 13.04 \%) ; \lambda_{\max }(\mathrm{DCM}) / \mathrm{nm} 230(\log \varepsilon 4.44), 283$ (4.54), 331 (4.39), 375 (4.21); $v_{\max }(\mathrm{Drift}) / \mathrm{cm}^{-1} 3398 \mathrm{~s}, 3373 \mathrm{~s}$, and 3325s (Ar NH), 3092w, and 3025w (Ar CH), $2956 \mathrm{~m}, 2928 \mathrm{~s}, 2870 \mathrm{~m}$ and $2856 \mathrm{~m}\left(\mathrm{CH}_{2}\right.$ and $\left.\mathrm{CH}_{3}\right), 1618 \mathrm{~m}, 1597 \mathrm{~s}, 1569 \mathrm{~s}, 1520 \mathrm{~s}, 1508 \mathrm{~s}, 1486 \mathrm{~s}$, $1459 \mathrm{~s}, 1436 \mathrm{~m}, 1408 \mathrm{~s}, 1340 \mathrm{~s}, 1322 \mathrm{~s}, 1284 \mathrm{~s}, 1247 \mathrm{~s}, 1202 \mathrm{~m}, 1160 \mathrm{~m}, 1121 \mathrm{w}, 1104 \mathrm{~m}, 1067 \mathrm{~m}$, $1043 \mathrm{w}, 926 \mathrm{w}, 897 \mathrm{~m}, 829 \mathrm{~m}, 820 \mathrm{~m}, 810 \mathrm{~m}, 753 \mathrm{~m}, 742 \mathrm{~m}, 627 \mathrm{w} ; \delta_{\mathrm{H}}\left(500 \mathrm{MHz} ; \mathrm{DMSO}-d_{6}\right) 9.39$ (2H, s, NH), 8.98 (1H, s, H-3), 7.48 (2H, s, PhNH), 7.12-7.07 (6H, m, Ar H), 6.95 (4H, d, J 8.1 $\mathrm{Hz}, \operatorname{Ar} \mathbf{H}), 6.82-6.79$ (2H, m, Ar H), $6.71(4 \mathrm{H}, \mathrm{d}, J 8.2 \mathrm{~Hz}, \operatorname{Ar} \mathbf{H}), 5.97$ (1H, s, H-6), 2.45 (4H, t, $J$ 7.7 Hz, Ar $\mathrm{CH}_{2}$ ), 1.50-1.44 (4H, quintet, $\mathrm{CH}_{2}$ ), 1.30-1.23 (4H, sextet, $\left.\mathrm{CH}_{2}\right), 0.87$ (6H, t, $J$ $\left.7.6 \mathrm{~Hz}, \mathrm{CH}_{3}\right)$; one carbon signal missing $\delta_{\mathrm{C}}\left(125 \mathrm{MHz}\right.$; DMSO- $\left.d_{6}\right) 146.69(\mathrm{Ar} \mathrm{CNH}), 140.21(\mathrm{Ar}$ CNH), 139.94 (Ar CNH), 134.79 (Ar CNH), 128.64, 127.53, 127.38, 125.93, 125.01, 119.84, 
119.02, 116.88, $94.97(\mathbf{C H}-3), 34.17\left(\mathrm{Ar} \mathbf{C H}_{2}\right), 33.32\left(\mathbf{C H}_{2}\right), 21.68\left(\mathbf{C H}_{2}\right), 13.77\left(\mathbf{C H}_{3}\right) ; \mathrm{m} / z$ (FAB) $644\left(\mathrm{M}^{+}, 100 \%\right)$ (Found: $\mathrm{M}^{+}, 644.3121 . \mathrm{C}_{38} \mathrm{H}_{40} \mathrm{~N}_{6} \mathrm{O}_{2}$ requires $\left.M, 644.3111\right)$.

1,5-Bis[ $N$-( $N$ '-4-t-butylphenyl-1,2-benzenediamino)]-2,4-dinitrobenzene (7c). Similarly (Method 2) the treatment of 1,5-difluoro-2,4-dinitrobenzene 6 with $N$-(4-t-butylphenyl)-1,2benzenediamine 5c and Hünig's base gave the title compound 7c $(87 \%)$ as a brick red precipitate, mp 194.5-198 C (from EtOH) (Found: $\mathrm{C}, 71.04 ; \mathrm{H}, 6.33 ; \mathrm{N}, 13.16 . \mathrm{C}_{38} \mathrm{H}_{40} \mathrm{~N}_{6} \mathrm{O}_{4}$ requires $\mathrm{C}, 70.81 ; \mathrm{H}, 6.21 ; \mathrm{N}, 13.04 \%) ; \lambda_{\max }(\mathrm{DCM}) / \mathrm{nm} 231$ (log $\varepsilon$ 4.43), 285 (4.61), 331 (4.48), $365 \inf (4.28) ; v_{\max }(\mathrm{Drift}) / \mathrm{cm}^{-1} 3355 \mathrm{~s}$ and 3311s (Ar NH), 3095w, 3071w and 3055w (Ar CH), 2963s, 2904s and 2869s $\left(\mathrm{CH}_{3}\right), 1636 \mathrm{~s}, 1605 \mathrm{~s}, 1540 \mathrm{~s}, 1508 \mathrm{~s}, 1488 \mathrm{~s}, 1439 \mathrm{~s}, 1424 \mathrm{~s}, 1363 \mathrm{~s}, 1349 \mathrm{~s}$, $1307 \mathrm{~s}, 1243 \mathrm{~s}, 1206 \mathrm{~m}, 1126 \mathrm{~m}, 1111 \mathrm{~m}, 1069 \mathrm{~m}, 1020 \mathrm{~m}, 950 \mathrm{w}, 931 \mathrm{~m}, 877 \mathrm{w}, 843 \mathrm{~m}, 829 \mathrm{~m}, 745 \mathrm{w}$; $\delta_{\mathrm{H}}\left(400 \mathrm{MHz} ; \mathrm{CD}_{2} \mathrm{Cl}_{2}\right) 9.33(2 \mathrm{H}, \mathrm{s}, \mathrm{NH}), 9.26(1 \mathrm{H}, \mathrm{s}, \mathbf{H}-3), 7.23(4 \mathrm{H}, \mathrm{d}, J 8.6 \mathrm{~Hz}, \mathrm{Ar} \mathbf{H}), 7.15-$ 7.14 (4H, m, Ar H), 7.09 (2H, d, J 7.7 Hz, Ar H), 6.86-6.81 (2H, m, Ar H), 6.78 (4H, d, J 8.6 $\mathrm{Hz}, \operatorname{Ar} \mathbf{H}), 5.98(1 \mathrm{H}, \mathrm{s}, \mathbf{H}-6), 5.63(2 \mathrm{H}, \mathrm{s}, \mathrm{PhNH}), 1.27\left(18 \mathrm{H}, \mathrm{s}, \mathrm{CH}_{3}\right) ; \delta_{\mathrm{C}}\left(50 \mathrm{MHz} ; \mathrm{CD}_{2} \mathrm{Cl}_{2}\right)$ 148.02 (Ar CNH), 146.05 (Ar CNH), 141.01 (Ar CNH), 139.51 (Ar CNH), 129.19, 128.88, 128.02, 126.65, 126.18, 125.62, 120.94, 120.26, 116.93, 96.87 (CH-3), 34.67 (CMe3), 31.72 $\left(\mathrm{CH}_{3}\right) ; m / z(\mathrm{EI}) 644\left(\mathrm{M}^{+}, 5 \%\right)$ (Found: $\mathrm{M}^{+}, 644.3117 . \mathrm{C}_{38} \mathrm{H}_{40} \mathrm{~N}_{6} \mathrm{O}_{4}$ requires $\left.M, 644.3111\right)$.

1,5-Bis[ $N$-( $N^{\prime}$-n-butyl-1,2-benzenediamino)]-2,4-dinitrobenzene (7d). Similarly the treatment of 1,5-difluoro-2,4-dinitrobenzene $\mathbf{6}$ with $N$-(n-butyl)-1,2-benzenediamine $5 \mathbf{d}$ and Hünig's base gave the title compound 7d (83\%) as bright red prisms, mp 144-147 C (from EtOH) (Found: C, 63.35; $\mathrm{H}, 6.44 ; \mathrm{N}, 17.33 . \mathrm{C}_{26} \mathrm{H}_{32} \mathrm{~N}_{6} \mathrm{O}_{4}$ requires $\left.\mathrm{C}, 63.41 ; \mathrm{H}, 6.50 ; \mathrm{N}, 17.07 \%\right) ; \lambda_{\max }(\mathrm{DCM}) / \mathrm{nm}$ 230 (log $\varepsilon$ 4.46), 244 (4.46), 331 (4.42), $365 \mathrm{inf}$ (4.25); $v_{\max }(\mathrm{Drift}) / \mathrm{cm}^{-1} 3413 \mathrm{~m}$ and 3379s (Ar $\mathrm{NH}), 3103 \mathrm{w}, 3074 \mathrm{w}$ and 3043w $(\mathrm{Ar} \mathrm{CH}), 2956 \mathrm{~s}, 2931 \mathrm{~s}$ and $2870 \mathrm{~m}\left(\mathrm{CH}_{2}\right.$ and $\left.\mathrm{CH}_{3}\right), 1602 \mathrm{~s}$, $1583 \mathrm{~s}, 1523 \mathrm{~s}, 1460 \mathrm{~m}, 1431 \mathrm{~m}, 1412 \mathrm{~m}, 1379 \mathrm{w}, 1342 \mathrm{~m}, 1327 \mathrm{~m}, 1302 \mathrm{~m}, 1265 \mathrm{~m}, 1223 \mathrm{~m}, 1190 \mathrm{~m}$, $1159 \mathrm{w}, 1103 \mathrm{w}, 1072 \mathrm{w}, 1043 \mathrm{w}, 989 \mathrm{w}, 924 \mathrm{w}, 831 \mathrm{w}, 744 \mathrm{w}, 690 \mathrm{w}, 634 \mathrm{w} ; \delta_{\mathrm{H}}\left(200 \mathrm{MHz} ; \mathrm{CD}_{2} \mathrm{Cl}_{2}\right)$ $9.24(1 \mathrm{H}, \mathrm{s}, \mathbf{H}-3), 9.21$ (2H, br s, NH), $7.13(2 \mathrm{H}, \mathrm{dd}, J 7.7,7.6 \mathrm{~Hz}, \mathrm{Ar} \mathbf{H}), 6.99(2 \mathrm{H}, \mathrm{d}, J 7.4 \mathrm{~Hz}$, Ar H), 6.63-6.56 (4H, m, Ar H), $5.73(1 \mathrm{H}, \mathrm{s}, \mathbf{H}-6), 3.82$ (2H, br s, NH), 3.03-2.97 (4H, m, $\left.\mathrm{NCH}_{2}\right), 1.59-1.14\left(8 \mathrm{H}, \mathrm{m}, \mathrm{CH}_{2}\right), 0.93\left(6 \mathrm{H}, \mathrm{t}, J 7.1 \mathrm{~Hz}, \mathrm{CH}_{3}\right) ; \delta_{\mathrm{C}}\left(50 \mathrm{MHz} ; \mathrm{CD}_{2} \mathrm{Cl}_{2}\right) 148.14$, 144.76, 129.28 (Ar CH), 129.22 (Ar CH), 127.44 (Ar CH), 126.06, 122.57, 117.04 (Ar CH), 111.85 (Ar CH), 96.94 (Ar CH), $43.80\left(\mathrm{NCH}_{2}\right), 32.05\left(\mathrm{CH}_{2}\right), 20.86\left(\mathrm{CH}_{2}\right), 14.23\left(\mathrm{CH}_{3}\right) ; \mathrm{m} / z$ (FAB) $492\left(\mathrm{M}^{+}, 100 \%\right)$ (Found: $\mathrm{M}^{+}, 492.2491 . \mathrm{C}_{26} \mathrm{H}_{32} \mathrm{~N}_{6} \mathrm{O}_{4}$ requires $M$, 492.2485).

\section{Preparation of unsymmetrical derivative}

\section{1-[N-( $N^{\prime}$-t-Butylphenyl-1,2-benzenediamino $\left.)\right]-5-\left[N\right.$-( $N^{\prime}$-phenyl-1,2-benzene-diamino $\left.)\right]-2,4-$}

dinitrobenzene (7e). To a stirred suspension of 1-[N-(N'-phenyl-1,2-benzenediamino $)]-5$-fluoro2,4-dinitrobenzene $9(1.37 \mathrm{~g}, 3.72 \mathrm{mmol})$ in $\mathrm{EtOH}(50 \mathrm{~mL})$ at $c a .20 \mathrm{C}$, under argon, $N-(4-t$ butylphenyl)-1,2-benzenediamine $5 \mathrm{c}(2 \mathrm{~g}, 8.33 \mathrm{mmol})$ was added in one portion. The color of the reaction mixture became deep orange and the reaction mixture was heated under reflux for $24 \mathrm{~h}$, then allowed to cool to $c a .20 \mathrm{C}$. The red precipitate was filtered, washed with hot water, dried and recrystallised to afford the title compound $7 \mathbf{e}(1.99 \mathrm{~g}, 91 \%)$ as an orange powder, mp 179$185 \mathrm{C}$ (from EtOH) (Found: C, 69.70; H, 5.55; N, 14.15. $\mathrm{C}_{34} \mathrm{H}_{32} \mathrm{~N}_{6} \mathrm{O}_{4}$ requires $\mathrm{C}, 69.39 ; \mathrm{H}$, 
5.44; N, 14.29\%); $\lambda_{\max }(\mathrm{DCM}) / \mathrm{nm} 229$ (log $\varepsilon$ 4.42), 284 (4.54), 331 (4.40), 374 (4.21); $v_{\max }(\mathrm{Drift}) / \mathrm{cm}^{-1} 3411 \mathrm{~m}, 3382 \mathrm{~m}, 3337 \mathrm{~s}$ and $3327 \mathrm{~s},(\mathrm{Ar} \mathrm{NH}), 3064 \mathrm{w}$ and $3040 \mathrm{w}(\mathrm{Ar} \mathrm{CH}), 2962 \mathrm{~m}$, 2901w, 2866w, 1619s, 1598s, 1571s, 1518s, 1482s, 1465s, 1459s, 1409s, 1361m, 1339s, 1324s, $1313 \mathrm{~s}, 1287 \mathrm{~s}, 1247 \mathrm{~s}, 1204 \mathrm{~s}, 1191 \mathrm{~s}, 1158 \mathrm{~m}, 1123 \mathrm{w}, 1102 \mathrm{w}, 1068 \mathrm{~m}, 932 \mathrm{w}, 897 \mathrm{w}, 835 \mathrm{w}, 781 \mathrm{w}$, $740 \mathrm{~m}, 694 \mathrm{~m} ; \delta_{\mathrm{H}}\left(200 \mathrm{MHz} ; \mathrm{CD}_{2} \mathrm{Cl}_{2}\right) 9.39(1 \mathrm{H}, \mathrm{s}, \mathrm{NH}), 9.36(1 \mathrm{H}, \mathrm{s}, \mathrm{NH}), 9.14(1 \mathrm{H}, \mathrm{s}, \mathbf{H}-3), 7.29-$ $7.11(10 \mathrm{H}, \mathrm{m}, \mathrm{Ar} \mathbf{H}), 6.99-6.83(7 \mathrm{H}, \mathrm{m}, \operatorname{Ar} \mathbf{H}), 6.09(1 \mathrm{H}, \mathrm{s}, \mathbf{H}-6), 5.88(1 \mathrm{H}, \mathrm{s}, \mathrm{NH}), 5.81(1 \mathrm{H}, \mathrm{s}$, $\mathrm{NH}), 1.32\left(9 \mathrm{H}, \mathrm{s}, \mathrm{CH}_{3}\right)$; one peak missing $\delta_{\mathrm{C}}\left(50 \mathrm{MHz} ; \mathrm{CD}_{2} \mathrm{Cl}_{2}\right)$ 147.84, 147.72, 145.82, 142.35, 140.86, 140.14, 139.39, 129.68 (Ar CH), 128.98 (Ar CH), $128.70(\mathrm{Ar} \mathrm{CH}), 128.63(\mathrm{Ar} \mathrm{CH})$, 127.77 (Ar CH), 127.69 (Ar CH), 126.48 (Ar CH), 126.31, 125.93, 125.46, 122.37 (Ar CH), 121.48 (Ar CH), 120.75 ( $\mathrm{Ar} \mathrm{CH}), 120.09(\mathrm{Ar} \mathrm{CH}), 119.57(\mathrm{Ar} \mathrm{CH}), 117.68(\mathrm{Ar} \mathrm{CH}), 116.79(\mathrm{Ar}$ $\mathrm{CH}), 96.62(\mathrm{Ar} \mathrm{CH}), 34.51\left(\mathrm{CCH}_{3}\right), 31.58\left(\mathrm{CCH}_{3}\right) ; \mathrm{m} / z(\mathrm{FAB}) 588\left(\mathrm{M}^{+}, 100 \%\right)$ (Found: $\mathrm{M}^{+}$, 588.2486. $\mathrm{C}_{34} \mathrm{H}_{32} \mathrm{~N}_{6} \mathrm{O}_{4}$ requires $\left.M, 588.2485\right)$.

$N^{\prime}, N^{\prime \prime \prime \prime '-B i s[2-(~} N$-phenylbenzamino)]-1,2,4,5-benzenetetraamine (8a). To $\quad$ a $\quad$ stirred suspension of 1,5-bis[ $N$-( $N$ '-phenyl-1,2-benzenediamino) $]$-2,4-dinitrobenzene 7a $\quad(100 \mathrm{mg}$, $0.188 \mathrm{mmol})$ in EtOH $(20 \mathrm{~mL})$ at $c a \cdot 20^{\circ} \mathrm{C}$, under argon, $(10 \%)$ palladium on carbon $(100 \mathrm{mg})$ was added in one portion. The reaction mixture was evacuated (to $25 \mathrm{mmHg}$ ) and flushed with argon 3 times then the mixture was evacuated (to $25 \mathrm{mmHg}$ ) and flushed with hydrogen 3 times. The reaction mixture was then left to stir under an atmosphere of hydrogen. The color of the mixture became dark red and after $1 \mathrm{~h}$ the color disappeared and a cream colored precipitate was observed. The suspension was diluted with sufficient dichloromethane to dissolve the precipitate, which was filtered through a celite pad and all volatiles were removed to afford brown oil. This was diluted with cold EtOH and triturated to afford a crude specimen of the title compound 8a (80mg, 90\%) as brown needles. A sample of the crude product was further purified by dry flash chromatography on silica $\left(\mathrm{Et}_{2} \mathrm{O}, 100 \%\right)$ to give compound 8a as colorless needles, mp 127$130 \mathrm{C}$ starts to melt in this range, becoming green in color and further melting stops (from $\mathrm{Et}_{2} \mathrm{O}$ ) (Found: C, 76.60; H, 6.06; N, 17.90. $\mathrm{C}_{30} \mathrm{H}_{28} \mathrm{~N}_{4}$ requires $\mathrm{C}, 76.27 ; \mathrm{H}, 5.93 ; \mathrm{N}, 17.79 \%$ ); $\lambda_{\max }(\mathrm{DCM}) / \mathrm{nm} 241(\log \varepsilon 4.61), 300 \inf (4.32) ; v_{\max }(\mathrm{Drift}) / \mathrm{cm}^{-1} 3450 \mathrm{w}, 3415 \mathrm{w}, 3373 \mathrm{~m}, 3337 \mathrm{w}$ and $3277 \mathrm{~m}\left(\mathrm{NH}\right.$ and $\left.\mathrm{NH}_{2}\right), 3084 \mathrm{w}$ and $3047 \mathrm{w}(\mathrm{Ar} \mathrm{CH}), 1623 \mathrm{~m}, 1603 \mathrm{~s}, 1595 \mathrm{~s}, 1524 \mathrm{~s}, 1499 \mathrm{~s}$, $1470 \mathrm{~m}, 1459 \mathrm{~m}, 1443 \mathrm{~m}, 1424 \mathrm{~m}, 1347 \mathrm{~m}, 1319 \mathrm{~s}, 1296 \mathrm{~m}, 1258 \mathrm{~s}, 1203 \mathrm{w}, 1178 \mathrm{w}, 1152 \mathrm{w}, 1104 \mathrm{~m}$, $1042 \mathrm{~m}, 994 \mathrm{w}, 880 \mathrm{w}, 845 \mathrm{~m}, 798 \mathrm{w}, 756 \mathrm{~m}, 746 \mathrm{~m}, 693 \mathrm{~m}, 654 \mathrm{~m} ; \delta_{\mathrm{H}}\left(500 \mathrm{MHz} ; \mathrm{DMSO}-d_{6}\right) 7.21$ (2H, s, PhNH), $7.14(4 \mathrm{H}, \mathrm{dd}, J$ 7.4, $7.5 \mathrm{~Hz}, \mathrm{Ph} \mathbf{H}-3), 7.09\left(2 \mathrm{H}, \mathrm{d}, J 7.7 \mathrm{~Hz}, \mathrm{C}_{6} \mathrm{H}_{4} \mathrm{~N}_{2} \mathbf{H}-2\right.$ or 5), $6.84(2 \mathrm{H}, \mathrm{dd}, J 7.8,7.7 \mathrm{~Hz}, \mathrm{Ph} \mathbf{H}-4), 6.80(4 \mathrm{H}, \mathrm{d}, J 8.6 \mathrm{~Hz}, \mathrm{Ph} \mathbf{H}-2), 6.69(2 \mathrm{H}, \mathrm{dd}, J 7.3,7.5 \mathrm{~Hz}$, $\mathrm{C}_{6} \mathrm{H}_{4} \mathrm{~N}_{2} \mathbf{H}-3$ or 4), $6.61\left(2 \mathrm{H}, \mathrm{dd}, J 7.5,7.6 \mathrm{~Hz}, \mathrm{C}_{6} \mathrm{H}_{4} \mathrm{~N}_{2} \mathbf{H}-3\right.$ or 4$), 6.61\left(1 \mathrm{H}, \mathrm{s}, \mathrm{C}_{6} \mathrm{H}_{2} \mathrm{~N}_{4} \mathbf{H}-3\right.$ or 6$)$, $6.48\left(2 \mathrm{H}, \mathrm{d}, J 8.1 \mathrm{~Hz}, \mathrm{C}_{6} \mathrm{H}_{4} \mathrm{~N}_{2} \mathbf{H}-2\right.$ or 5), $6.21\left(1 \mathrm{H}, \mathrm{s}, \mathrm{C}_{6} \mathrm{H}_{2} \mathrm{~N}_{4} \mathbf{H}-3\right.$ or 6$), 5.98(2 \mathrm{H}, \mathrm{s}, \mathrm{NH}), 4.48$ $\left(4 \mathrm{H}, \mathrm{s}, \mathrm{NH}_{2}\right) ; \delta_{\mathrm{C}}\left(50 \mathrm{MHz} ; \mathrm{DMSO}-d_{6}\right) 146.14,142.92,141.92,129.15,128.79,125.64,124.27$, $123.13,118.12,117.25,116.78,114.98,113.07,101.27 ; \mathrm{m} / z$ (EI) $472\left(\mathrm{M}^{+}, 100 \%\right), 470\left(\mathrm{M}^{+}-2 \mathrm{H}\right.$, 90), $453\left(\mathrm{M}^{+}-2 \mathrm{H}-\mathrm{NH}_{3}, 20\right), 436\left(\mathrm{M}^{+}-2 \mathrm{H}-2 \mathrm{NH}_{2}, 45\right), 378$ (5), 361 (20), $287\left(\mathrm{M}^{+}-2 \mathrm{H}-\right.$ $\mathrm{PhNHC}_{6} \mathrm{H}_{4} \mathrm{NH}_{2}$, 95) (Found: $\mathrm{M}^{+}, 472.2383 . \mathrm{C}_{30} \mathrm{H}_{28} \mathrm{~N}_{6}$ requires $M$, 472.2375).

5,7-Diphenyl-5H,12H-quinoxalino[2,3-b]phenazine (3a). Method 1. To a stirred solution of $N^{\prime}, N^{\prime \prime \prime \prime \prime '-b i s[2-(~} N$-phenylbenzamino)]-1,2,4,5-benzenetetraamine 8a (444mg, $\left.0.94 \mathrm{mmol}\right)$ in EtOH 
$(20 \mathrm{~mL})$ at $c a .20 \mathrm{C}$, under an atmosphere of air, hydrochloric acid $(36 \%, 10 \mathrm{~mL})$ was added in one portion. The color of the reaction mixture became lilac, then blue. The reaction mixture was heated under reflux for $2 \mathrm{~h}$ then allowed to cool to $c a$. $20 \mathrm{C}$. A green-blue precipitate was observed and assumed to be the hydrochloride salt of compound 3a. The mixture was made basic (aq. $\mathrm{NaOH}$ ) and the precipitate was filtered, washed (hot water then cold ethanol) and dried to afford compound 3a (375mg, 91\%) as dark green needles, $\mathrm{mp}>365 \mathrm{C}$ dec. (from EtOH) (Found: $\mathrm{C}, 82.81 ; \mathrm{H}, 4.76 ; \mathrm{N}, 12.80 . \mathrm{C}_{30} \mathrm{H}_{20} \mathrm{~N}_{4}$ requires $\mathrm{C}, 82.57 ; \mathrm{H}, 4.59 ; \mathrm{N}, 12.84 \%$ ); $\lambda_{\max }(\mathrm{DCM}) / \mathrm{nm} 228$ (log $\varepsilon$ 4.26), 298 (4.98), 361 (3.90), 382 (4.09), 401 (4.50), 423 (4.79), 450 (3.90), 478 (4.02), 512 (3.84), 629 (4.15), 688 (4.41), 763 (4.41); $v_{\max }\left(\right.$ Drift) $/ \mathrm{cm}^{-1} 3052 \mathrm{~s}, 3026 \mathrm{~s}$ and $3007 \mathrm{~s}(\mathrm{Ar} \mathrm{CH}), 1615 \mathrm{w}, 1590 \mathrm{~m}, 1560 \mathrm{~s}, 1539 \mathrm{~m}, 1505 \mathrm{~s}, 1497 \mathrm{~s}, 1492 \mathrm{~s}, 1456 \mathrm{~s}, 1440 \mathrm{~s}, 1356 \mathrm{~s}$, $1333 \mathrm{~s}, 1318 \mathrm{~m}, 1308 \mathrm{~m}, 1250 \mathrm{~s}, 1227 \mathrm{w}, 1192 \mathrm{~m}, 1169 \mathrm{~m}, 1143 \mathrm{~m}, 1120 \mathrm{w}, 1071 \mathrm{w}, 1028 \mathrm{w}, 1004 \mathrm{w}$, 920w, 818m, 775m, 730s, 702m, 687m; ${ }^{1} \mathrm{H}$ NMR assignments supported by NOE experiment $\delta_{\mathrm{H}}\left(500 \mathrm{MHz} ; \mathrm{CD}_{2} \mathrm{Cl}_{2}\right)$ 7.42-7.39 (6H, m, Ph H'-3,4 and 5), 7.07-7.04 (6H, m, Ph H'-2,6 and $\left.\mathbf{H}-1\right)$, $6.94(2 \mathrm{H}, \mathrm{dd}, J 7.4,7.3 \mathrm{~Hz}, \mathbf{H}-2), 6.56(2 \mathrm{H}, \mathrm{dd}, J 7.5,7.6 \mathrm{~Hz}, \mathbf{H}-3), 6.16(1 \mathrm{H}, \mathrm{s}, \mathbf{H}-6), 6.05(2 \mathrm{H}$, d, $J 8.4 \mathrm{~Hz}, \mathbf{H}-4), 4.25(1 \mathrm{H}, \mathrm{s}, \mathbf{H}-13)$; the following carbon resonance's could be observed, $\delta_{\mathrm{C}}\left(125 \mathrm{MHz} ; \mathrm{CD}_{2} \mathrm{Cl}_{2}\right) 137.03,131.35,130.83,130.16,128.11,127.42,122.48,116.46 ; \mathrm{m} / \mathrm{z}$ (FAB) $437\left(\mathrm{MH}^{+}, 40 \%\right), m / z$ (EI) $436\left(\mathrm{M}^{+}, 100 \%\right), 359\left(\mathrm{M}^{+}-\mathrm{Ph}, 30\right)$ (Found: $\mathrm{M}^{+}, 436.1689$. $\mathrm{C}_{30} \mathrm{H}_{20} \mathrm{~N}_{4}$ requires $M, 436.1688$ ).

Method 2. A stirred solution of $N^{\prime}, N^{\prime \prime \prime \prime '-b i s[2-(~} N$-phenylbenzamino)]-1,2,4,5-benzenetetraamine 8a $(475 \mathrm{mg}, 1.01 \mathrm{mmol})$ in EtOH $(20 \mathrm{~mL})$, under an atmosphere of air, was heated under reflux. The color of the reaction mixture rapidly became brown then green and a green crystalline precipitate was formed within 20 min. TLC monitoring over a period of $7 \mathrm{~h}$ showed consumption of the starting material had ceased and so the precipitate was collected by filtration and dried to give $101.8 \mathrm{mg}$ of a green crystalline material. The filtrate was heated to reflux and rapidly precipitated a further $99.2 \mathrm{mg}$ which was removed by filtration. The process was repeated collecting a further four fractions $(64.1,38.0,49.1$ and $16.8 \mathrm{mg})$ until it became impractical to recover more precipitate. No further purification was required, the total recovery of compound 3a was $369 \mathrm{mg}, 84 \%$ yield; the sample was identical to that described above.

Method 3. To a stirred suspension of 1,5-bis[ $N$-( $N$ '-phenyl-1,2-benzenediamino $)]-2,4-$ dinitrobenzene 7a (100mg, $0.188 \mathrm{mmol})$ in EtOH $(20 \mathrm{~mL})$ at $c a .20 \mathrm{C}$, under argon, $(10 \%)$ palladium on carbon $(100 \mathrm{mg}$ ) was added in one portion. The reaction mixture was evacuated (to $25 \mathrm{mmHg}$ ) and flushed with argon 3 times, then evacuated (to $25 \mathrm{mmHg}$ ) and flushed with hydrogen 3 times. The reaction mixture was then left to stir under a hydrogen atmosphere. The color of the mixture became dark red and after $3 \mathrm{~h}$ this red color disappeared and a cream colored precipitate was observed. The mixture was heated gently to dissolve the precipitated amine and then hot-filtered through a short pad of celite to remove the palladium catalyst. The dark ethanolic solution of the amine was heated exposed to atmospheric oxygen until a green precipitate was formed. This was filtered off and the filtrate was taken to reflux until more precipitate was formed. The precipitate was removed and the process repeated until there was no 
further precipitate. Combining the precipitated material gave the title compound 3a (70mg, 85\%) identical to an authentic sample.

5,7-Bis(4-n-butylphenyl)-5H,12H-quinoxalino[2,3-b]phenazine (3b). Similarly (Method 3) treatment of 1,5-bis[ $N$-( $N^{\prime}$-4- $n$-butylphenyl-1,2-benzenediamino) $]$-2,4-dinitrobenzene $7 \mathbf{b}$ gave the title compound $\mathbf{3 b}(77 \%)$ as dark green needles, mp 310-320 C dec. (from EtOH) (Found: C, 83.38; H, 6.69; N, 9.98. $\mathrm{C}_{38} \mathrm{H}_{36}{ }^{\mathrm{N}} 4$ requires $\left.\mathrm{C}, 83.21 ; \mathrm{H}, 6.57 ; \mathrm{N}, 10.22 \%\right) ; \lambda_{\max }(\mathrm{DCM}) / \mathrm{nm} 228$ (log $\varepsilon$ 4.33), 299 (5.05), 333 (3.94), 359 (3.92), 382 (4.12), 401 (4.58), 423 (4.93), 450 (3.89), 478 (4.01), 512 (3.67), $629 \inf$ (3.96), 693 (4.37), 760 (4.50); $v_{\max }\left(\right.$ Drift) $/ \mathrm{cm}^{-1} 3052 \mathrm{~m}, 3044 \mathrm{~m}$ and $3032 \mathrm{~m}(\mathrm{Ar} \mathrm{CH}), 2999 \mathrm{~m}, 2948 \mathrm{~m}, 2927 \mathrm{~s}, 2870 \mathrm{~m}$ and $2857 \mathrm{~m}\left(\mathrm{CH}_{2}\right.$ and $\left.\mathrm{CH}_{3}\right), 1615 \mathrm{w}, 1602 \mathrm{w}$, $1592 \mathrm{w}, 1559 \mathrm{~s}, 1539 \mathrm{~m}, 1507 \mathrm{~s}, 1457 \mathrm{~s}, 1441 \mathrm{~s}, 1365 \mathrm{~m}, 1352 \mathrm{~s}, 1333 \mathrm{~s}, 1308 \mathrm{~s}, 1249 \mathrm{~m}, 1227 \mathrm{w}$, $1194 \mathrm{~m}, 1178 \mathrm{~m}, 1141 \mathrm{~m}, 1115 \mathrm{w}, 1081 \mathrm{w}, 1022 \mathrm{w}, 972 \mathrm{w}, 918 \mathrm{w}, 836 \mathrm{w}, 823 \mathrm{~m}, 816 \mathrm{~m}, 770 \mathrm{w}, 731 \mathrm{~m}$, 672w, 642w, 608m, 589w; $\delta_{\mathrm{H}}\left(500 \mathrm{MHz} ; \mathrm{CD}_{2} \mathrm{Cl}_{2}\right) 7.24(4 \mathrm{H}, \mathrm{d}, J 8.2 \mathrm{~Hz}, \mathrm{~N}-\mathrm{Ar} \mathrm{H}), 7.02(2 \mathrm{H}, \mathrm{d}, J$ $7.9 \mathrm{~Hz}, \mathbf{H}-1), 6.97$ (4H, d, J 8.2 Hz, N-Ar H), 6.91 (2H, dd, J 7.6, 7.5 Hz, H-2), 6.54 (2H, dd, J 7.6, 7.7 Hz, H-3), 6.15 (1H, s, H-6), 6.04 (2H, d, J 7.7 Hz, H-4), 4.47 (1H, s, H-13), 2.62 (4H, t, $\left.J 7.8 \mathrm{~Hz}, \operatorname{Ar} \mathrm{CH}_{2}\right), 1.60$ (4H, m, $\left.\mathrm{CH}_{2}\right), 1.43\left(4 \mathrm{H}, \mathrm{m}, \mathrm{CH}_{2}\right), 1.01(6 \mathrm{H}, \mathrm{t}, J 7.3 \mathrm{~Hz}, \mathrm{CH}$ ); $\delta_{\mathrm{C}}\left(125 \mathrm{MHz} ; \mathrm{CD}_{2} \mathrm{Cl}_{2}\right) 150.94,145.46,144.90,144.21,134.50,131.08,131.04,127.83,127.25$, 125.97, 122.86, 116.54, 103.64 (Ar C-13), 93.03 (Ar C-6), $35.89\left(\mathrm{Ar} \mathbf{C H}_{2}\right), 34.16\left(\mathbf{C H}_{2}\right), 23.12$ $\left(\mathrm{CH}_{2}\right), 14.27\left(\mathrm{CH}_{3}\right) ; \mathrm{m} / z$ (FAB) $549\left(\mathrm{MH}^{+}, 100 \%\right)$ (Found: $\mathrm{MH}^{+}, 549.3027 . \mathrm{C}_{38} \mathrm{H}_{37} \mathrm{~N}_{4}$ requires MH, 549.3018).

5,7-Bis(4-t-butylphenyl)-5H,12H-quinoxalino[2,3-b]phenazine (3c). Similarly (Method 3) treatment of 1,5-bis[ $N$-( $N^{\prime}$-4-t-butylphenyl-1,2-benzenediamino) $]$-2,4-dinitrobenzene 7c gave the title compound 3c (84\%) as dark green needles, mp 350-360 C dec. (from EtOH) (Found: C, 83.49; H, 6.72; N, 10.12. $\mathrm{C}_{38} \mathrm{H}_{36} \mathrm{~N}_{4}$ requires $\left.\mathrm{C}, 83.21 ; \mathrm{H}, 6.57 ; \mathrm{N}, 10.22 \%\right) ; \lambda_{\max }(\mathrm{DCM}) / \mathrm{nm} 227$ (log $\varepsilon$ 4.23), 298 (4.97), 363 (3.99), 401 (4.49), 423 (4.82), 450 (3.85), 478 (3.96), 512 (3.73), $580 \inf (3.78), 628$ (4.12), 688 (4.35), 763 (4.35); $v_{\max }\left(\right.$ Drift) $/ \mathrm{cm}^{-1} 3068 \mathrm{w}, 3051 \mathrm{w}$ and 3029w (Ar $\mathrm{CH}), 2965 \mathrm{~s}, 2902 \mathrm{~m}$ and $2870 \mathrm{~m}\left(\mathrm{CH}_{3}\right), 1615 \mathrm{w}, 1588 \mathrm{~m}, 1559 \mathrm{~s}, 1530 \mathrm{~m}, 1507 \mathrm{~s}, 1473 \mathrm{~m}, 1459 \mathrm{~s}$, $1440 \mathrm{~s}, 1406 \mathrm{~m}, 1395 \mathrm{~m}, 1365 \mathrm{~s}, 1354 \mathrm{~s}, 1332 \mathrm{~s}, 1310 \mathrm{~s}, 1267 \mathrm{~m}, 1249 \mathrm{~m}, 1217 \mathrm{~m}, 1201 \mathrm{~m}, 1191 \mathrm{~m}$, $1148 \mathrm{~m}, 1140 \mathrm{~m}, 1114 \mathrm{w}, 1033 \mathrm{w}, 1017 \mathrm{~m}, 930 \mathrm{w}, 848 \mathrm{w}, 832 \mathrm{~m}, 823 \mathrm{~m}, 741 \mathrm{~s}, 727 \mathrm{~m}, 649 \mathrm{w}, 608 \mathrm{~m}$; $\delta_{\mathrm{H}}\left(400 \mathrm{MHz} ; \mathrm{CD}_{2} \mathrm{Cl}_{2}\right) 7.46(4 \mathrm{H}, \mathrm{d}, J 8.4 \mathrm{~Hz}, \mathrm{~N}-\mathrm{Ar} \mathbf{H}), 7.04-7.00(6 \mathrm{H}, \mathrm{m}, \mathrm{N}-\mathrm{Ar} \mathbf{H}$ and H-1), 6.92 (2H, dd, J 7.6, 7.5 Hz, H-2), 6.52 (2H, dd, J 7.1, 7.2 Hz, H-3), 6.19 (1H, s, H-6), 5.87 (2H, d, $J$ $7.8 \mathrm{~Hz}, \mathbf{H}-4), 4.78(1 \mathrm{H}, \mathrm{s}, \mathbf{H}-13), 1.32\left(18 \mathrm{H}, \mathrm{s}, \mathrm{CH}_{3}\right) ; \delta_{\mathrm{C}}\left(100 \mathrm{MHz} ; \mathrm{CD}_{2} \mathrm{Cl}_{2}\right)$ 153.58, 150.70, $144.72,143.99,134.11,131.21,127.91,127.38,127.10,125.70,122.58,116.46,103.79$ (Ar C13), 92.46 (Ar C-6), $35.11\left(\mathrm{Cme}_{3}\right), 31.45\left(\mathrm{CH}_{3}\right) ; \mathrm{m} / z$ (EI) $548\left(\mathrm{M}^{+}, 13 \%\right)$ (Found: $\mathbf{M}^{+}, 548.2937$. $\mathrm{C}_{38} \mathrm{H}_{36} \mathrm{~N}_{4}$ requires $\left.M, 548.2940\right)$.

5,7-Bis(n-butyl)-5H,12H-quinoxalino[2,3-b]phenazine (3d). Similarly (Method 3) treatment of 1,5-bis[ $N$-( $N$-n-butyl-1,2-benzenediamino)]-2,4-dinitrobenzene 7d gave the title compound 3d $(80 \%)$ as dark green needles, mp 245-250 C dec. (from EtOH) (Found: C, 78.95; H, 7.02; N, 14.19. $\mathrm{C}_{26} \mathrm{H}_{28} \mathrm{~N}_{4}$ requires $\left.\mathrm{C}, 78.79 ; \mathrm{H}, 7.07 ; \mathrm{N}, 14.14 \%\right) ; \lambda_{\max }(\mathrm{DCM}) / \mathrm{nm} 228(\log \varepsilon 4.11), 294$ (4.82), 332 (3.75), $360 \inf$ (3.88), $380 \inf$ (4.07), 395 (4.50), 417 (4.77), 445 (3.76), 473 (3.95), 506 (3.69), $570 \inf$ (3.73), $631 \inf$ (4.03), 694 (4.30), 768 (4.38); $v_{\max }\left(\right.$ Drift) $/ \mathrm{cm}^{-1} 3047 \mathrm{~m}$ and 
3030m (Ar CH), 2958s, 2931s and 2866m $\left(\mathrm{CH}_{2}\right.$ and $\left.\mathrm{CH}_{3}\right), 1612 \mathrm{w}, 1562 \mathrm{~s}, 1541 \mathrm{~m}, 1516 \mathrm{~s}, 1487 \mathrm{~s}$, $1460 \mathrm{~s}, 1444 \mathrm{~s}, 1411 \mathrm{w}, 1356 \mathrm{~s}, 1300 \mathrm{~m}, 1246 \mathrm{~m}, 1226 \mathrm{~m}, 1196 \mathrm{w}, 1151 \mathrm{w}, 1134 \mathrm{w}, 1124 \mathrm{~m}, 1063 \mathrm{w}$, $1032 \mathrm{w}, 976 \mathrm{w}, 918 \mathrm{w}, 885 \mathrm{~m}, 852 \mathrm{w}, 804 \mathrm{~m}, 781 \mathrm{w}, 731 \mathrm{~s}, 696 \mathrm{w}, 600 \mathrm{~m} ; \delta_{\mathrm{H}}\left(500 \mathrm{MHz} ; \mathrm{CD}_{2} \mathrm{Cl}_{2}\right) 6.92$ (4H, d, J 8.1 Hz, H-1), 6.86 (4H, dd, J 7.0, $7.1 \mathrm{~Hz}, \mathbf{H}-2), 6.71(4 \mathrm{H}, \mathrm{d}, J 8.1 \mathrm{~Hz}, \mathbf{H}-4), 6.62$ (4H, dd, J 6.3, 6.4 Hz, H-3), 5.95 (1H, s, H-13), 5.60 (1H, s, H-6), 3.86 (4H, t, J 8.2 Hz, NCH $), 1.70-$ 1.65 (4H, quintet, $\left.\mathrm{CH}_{2}\right)$, 1.54-1.50 (4H, hextet, $\left.\mathrm{CH}_{2}\right), 1.04\left(6 \mathrm{H}, \mathrm{t}, J 7.4 \mathrm{~Hz}, \mathrm{CH}_{3}\right) ; \delta_{\mathrm{C}}(125 \mathrm{MHz}$; $\mathrm{CD}_{2} \mathrm{Cl}_{2}$ ) 150.65, 145.03, 142.66, 129.10, 126.97, 126.14, 123.15, 114.62, 103.28 (Ar C-13), 88.94 (Ar C-6), $46.47\left(\mathrm{NCH}_{2}\right), 28.43\left(\mathrm{CH}_{2}\right), 20.82\left(\mathrm{CH}_{2}\right), 14.16\left(\mathrm{CH}_{3}\right) ; \mathrm{m} / z(\mathrm{FAB}) 397\left(\mathrm{MH}^{+}\right.$, $100 \%$ ) (Found: $\mathrm{MH}^{+}, 397.2390 . \mathrm{C}_{26} \mathrm{H}_{29} \mathrm{~N}_{4}$ requires $M H, 397.2392$ ).

5-(4-t-Butylphenyl)-7-phenyl-5H,12H-quinoxalino[2,3-b]phenazine (3e). Similarly (Method $3)$ treatment of 1-[N-( $N^{\prime}-t$-butylphenyl-1,2-benzenediamino $\left.)\right]-5-\left[N-\left(N^{\prime}-\right.\right.$ phenyl-1,2benzenediamino)]-2,4-dinitrobenzene $7 \mathbf{e}$ gave the title compound $3 \mathbf{e}(58 \%)$ as dark green needles, mp 345-350 C dec. (from EtOH) (Found: $\mathrm{C}, 82.91 ; \mathrm{H}, 5.81 ; \mathrm{N}, 11.35 . \mathrm{C}_{34} \mathrm{H}_{28} \mathrm{~N}_{4}$ requires C, 82.93; H, 5.69; N, 11.38\%); $\lambda_{\max }(\mathrm{DCM}) / \mathrm{nm} 228$ (log $\varepsilon$ 4.33), 298 (4.98), 382 inf (4.11), 401 (4.50), 423 (4.81), 450 (3.90), 478 (4.01), 512 (3.84), 578 inf (3.90), 629 (4.16), 687 (4.41), 760 (4.40); $v_{\max }(\mathrm{Drift}) / \mathrm{cm}^{-1} 3064 \mathrm{w}$ and 3024w (Ar CH), 2966m, 2904w and $2872 \mathrm{w}\left(\mathrm{CH}_{3}\right), 1615 \mathrm{w}$, $1590 \mathrm{w}, 1562 \mathrm{~s}, 1537 \mathrm{~m}, 1508 \mathrm{~s}, 1461 \mathrm{~m}, 1442 \mathrm{~m}, 1399 \mathrm{w}, 1367 \mathrm{~m}, 1352 \mathrm{~s}, 1334 \mathrm{~s}, 1308 \mathrm{~s}, 1250 \mathrm{~m}$, $1196 \mathrm{~m}, 1173 \mathrm{~m}, 1142 \mathrm{~m}, 1123 \mathrm{w}, 1072 \mathrm{w}, 1027 \mathrm{w}, 973 \mathrm{w}, 924 \mathrm{w}, 832 \mathrm{~m}, 823 \mathrm{~m}, 815 \mathrm{~m}, 767 \mathrm{~m}, 731 \mathrm{~s}$, $702 \mathrm{~m}, 687 \mathrm{w}, 649 \mathrm{w}, 605 \mathrm{~m} ; \delta_{\mathrm{H}}\left(500 \mathrm{MHz} ; \mathrm{CD}_{2} \mathrm{Cl}_{2}\right) 7.46-7.42(4 \mathrm{H}, \mathrm{m}, \mathrm{N}-\mathrm{Ar} \mathrm{H}), 7.38(1 \mathrm{H}, \mathrm{dd}, J$ 9.2, 8.9 Hz, Ph H'-4), 7.13 (2H, d, J 8.9 Hz, N-Ar H), 7.07-7.00 (4H, m, N-Ar H, H-1 and H11), 6.91-6.86 (2H, m, H-2 and H-10), 6.56-6.50 (2H, m, H-3 and H-9), 6.17 (1H, s, H-6), 6.10 $(1 \mathrm{H}, \mathrm{d}, J 9.2 \mathrm{~Hz}, \mathbf{H}-11), 6.00(1 \mathrm{H}, \mathrm{d}, J 9.2 \mathrm{~Hz}, \mathbf{H}-4), 4.37$ (1H, s, H-13), 1.34 (9H, s, CH$)$; observable carbon signals, $\delta_{\mathrm{C}}\left(125 \mathrm{MHz} ; \mathrm{CD}_{2} \mathrm{Cl}_{2}\right)$ 153.78, 150.56, 144.49, 144.21, 144.09, $137.05,134.14,131.31,130.91,130.85,130.27,128.28,128.21,127.44,127.34,127.29,125.84$, 125.78, 123.13, 123.09, 116.62, 116.40, 103.62 (Ar C-13), 93.10 (Ar C-6), 35.30 (Cme $), 31.64$ $\left(\mathrm{CH}_{3}\right) ; m / z$ (FAB) $493\left(\mathrm{MH}^{+}, 100 \%\right)$ (Found: $\mathrm{MH}^{+}, 493.2385 . \mathrm{C}_{34} \mathrm{H}_{29} \mathrm{~N}_{4}$ requires $M H$, 493.2392).

12-Methyl-5,7-diphenyl-5H,12H-quinoxalino[2,3-b]phenazinium iodide (10). To a stirred solution of 5,7-diphenyl-5H,12H-quinoxalino[2,3-b]phenazine 3a $(22.5 \mathrm{mg}, 0.0516 \mathrm{mmol})$ in DCM $(7 \mathrm{~mL})$, at $c a .20 \mathrm{C}$, under argon, was added a large excess of iodomethane $(0.5 \mathrm{~mL})$ in one portion. After $2 \mathrm{~h}$ the color of the reaction mixture had changed from green to blue. Dilution with hexane afforded a hygroscopic precipitate which was dried to give compound 10 (25mg, 84\%) as blue crystals with a bronze luster, mp 180-190 C dec. (from 1,2-dichloroethane/pentane) (Found: $\mathrm{C}, 64.28 ; \mathrm{H}, 4.22 ; \mathrm{N}, 9.16 . \mathrm{C}_{31} \mathrm{H}_{23} \mathrm{IN}_{4}$ requires $\left.\mathrm{C}, 64.36 ; \mathrm{H}, 3.98 ; \mathrm{N}, 9.69 \%\right) ; \lambda_{\max }(\mathrm{DCM}) / \mathrm{nm} 229$ (log $\varepsilon$ 4.41), 290 (4.94), 360 (3.96), 485 (3.56), 517 (3.85), 561 (4.23), 605 (4.60), 659 (4.70); $\lambda_{\max }(\mathrm{EtOH}) / \mathrm{nm} 201(\log \varepsilon$ 4.67), 230 inf (4.48), 289 (4.88), 360 (3.80), 485 (3.50), 515 inf (3.80), 557 (4.15), 600 (4.50), 652 (4.56); $v_{\max }(\mathrm{Drift}) / \mathrm{cm}^{-1} 3068 \mathrm{w}, 3050 \mathrm{w}$ and $3021 \mathrm{w}(\mathrm{Ar} \mathrm{CH})$, $2963 \mathrm{w}\left(\mathrm{CH}_{3}\right), 1596 \mathrm{~m}, 1587 \mathrm{~m}, 1570 \mathrm{~s}, 1538 \mathrm{~m}, 1516 \mathrm{~s}, 1489 \mathrm{~s}, 1473 \mathrm{~s}, 1461 \mathrm{~s}, 1449 \mathrm{~m}, 1423 \mathrm{~m}$, $1380 \mathrm{~m}, 1354 \mathrm{w}, 1324 \mathrm{~m}, 1244 \mathrm{~m}, 1233 \mathrm{~m}, 1170 \mathrm{~m}, 1153 \mathrm{~m}, 1125 \mathrm{w}, 1071 \mathrm{w}, 1027 \mathrm{w}, 1001 \mathrm{w}, 966 \mathrm{w}$, $831 \mathrm{w}, 777 \mathrm{w}, 756 \mathrm{~m}, 704 \mathrm{w}, 688 \mathrm{~m} ; \delta_{\mathrm{H}}\left(500 \mathrm{MHz} ; \mathrm{CD}_{2} \mathrm{Cl}_{2}\right) 7.93(1 \mathrm{H}, \mathrm{d}, J 8.2 \mathrm{~Hz}, \mathrm{Ar} \mathbf{H}), 7.60-7.50$ 
(7H, m, Ar and $\mathrm{Ph} \mathbf{H}), 7.45(1 \mathrm{H}, \mathrm{dd}, J$ 7.6, 7.4 Hz, $\mathrm{Ar} \mathbf{H}), 7.19-7.17(5 \mathrm{H}, \mathrm{m}, \mathrm{Ar}$ and $\mathrm{Ph} \mathbf{H}), 7.13$ $(1 \mathrm{H}, \mathrm{d}, J 8.1 \mathrm{~Hz}, \operatorname{Ar} \mathbf{H}), 6.86-6.83(3 \mathrm{H}, \mathrm{m}, \mathrm{Ar} \mathbf{H}), 6.29(1 \mathrm{H}, \mathrm{d}, J 8.0 \mathrm{~Hz}, \mathrm{Ar} \mathbf{H}), 4.72(1 \mathrm{H}, \mathrm{s}, \mathrm{Ar}$ H), $3.58\left(3 \mathrm{H}, \mathrm{s}, \mathrm{CH}_{3}\right) ; \delta_{\mathrm{C}}\left(125 \mathrm{MHz} ; \mathrm{CD}_{2} \mathrm{Cl}_{2}\right) 149.44,148.70,142.18,140.39,138.86,136.26$, 135.47, 133.11, 132.21 ( $\mathrm{Ph} \mathrm{CH}), 132.07,131.80$ (Ph CH), 131.68, 131.15, 131.07, 131.02, 130.23, 129.28, 128.25 ( $\mathrm{Ph} \mathrm{CH}), 128.04,127.24(\mathrm{Ph} \mathrm{CH}), 124.65,118.25,117.75,115.16$, 104.02, 95.07, $35.27\left(\mathrm{CH}_{3}\right) ; \mathrm{m} / z$ (EI) $451\left(\mathrm{M}^{+}-\mathrm{I}, 45 \%\right), 128$ (HI, 100) (Found: $\mathrm{M}^{+}$-I, 451.1918. $\mathrm{C}_{31} \mathrm{H}_{23} \mathrm{~N}_{4}$ requires $\left.M-I, 451.1923\right)$.

12,14-Dihydro-5,7-diphenyl-5H,12H-quinoxalino[2,3-b]phenazinium bisperchlorate (11). To a suspension of 5,7-diphenyl-5H,12H-quinoxalino[2,3-b]phenazine 3a (192mg, $0.44 \mathrm{mmol})$ in acetonitrile $(50 \mathrm{~mL})$, at $c a .20 \mathrm{C}$, was added $(60 \%)$ aqueous perchloric acid $(1 \mathrm{~mL})$. The color of the mixture turned deep blue and the suspension dissolved. The mixture was filtered through a celite pad and the filtrate was then diluted with ether until a cloudy suspension had formed. On standing this formed crystals and filtration gave the title compound $\mathbf{1 1}(236 \mathrm{mg}, 88 \%)$ as a golden precipitate, $\mathrm{mp}>375 \mathrm{C}$ (from acetonitrile/ether) (Found: $\mathrm{C}, 56.58 ; \mathrm{H}, 3.76 ; \mathrm{N}, 8.80$. $\mathrm{C}_{30} \mathrm{H}_{22} \mathrm{Cl}_{2} \mathrm{~N}_{4} \mathrm{O}_{8}$ requires $\left.\mathrm{C}, 56.60 ; \mathrm{H}, 3.46 ; \mathrm{N}, 8.81 \%\right) ; \lambda_{\max }(\mathrm{EtOH}) / \mathrm{nm} 206(\log \varepsilon$ 4.95), $225 \mathrm{inf}$ (4.33), 289 (5.02), 365 (4.01), $480 \inf$ (3.75), 516 (3.96), 561 (4.21), 606 (4.58), 660 (4.68); $\lambda_{\max }\left[\mathrm{EtOH} /\left(\right.\right.$ aq.) $\left.\mathrm{HclO}_{4}\right] / \mathrm{nm} 206$ (log \& 4.93), 225 (4.41), 291 (4.90), 370 (3.79), $510 \inf (3.80)$, $538(4.18), 585$ (4.63), 637 (5.01); $v_{\max }(\mathrm{Drift}) / \mathrm{cm}^{-1} 3609 \mathrm{w}, 3587 \mathrm{w}$ and $3567 \mathrm{w}(\mathrm{NH}), 3146 \mathrm{w}$, $3122 \mathrm{w}$, and $3075 \mathrm{w}(\mathrm{Ar} \mathrm{CH}), 1616 \mathrm{~s}, 1558 \mathrm{~s}, 1541 \mathrm{~s}, 1519 \mathrm{~s}, 1508 \mathrm{~s}, 1474 \mathrm{~s}, 1457 \mathrm{~m}, 1345 \mathrm{~m}, 1316 \mathrm{~m}$, $1249 \mathrm{~s}, 1161 \mathrm{~s}, 1100 \mathrm{~s}\left(\mathrm{ClO}_{4}\right), 1004 \mathrm{w}, 977 \mathrm{w}, 931 \mathrm{w}, 836 \mathrm{w}, 773 \mathrm{~m}, 697 \mathrm{~m}, 686 \mathrm{~m}, 625 \mathrm{~m}$; $\delta_{\mathrm{H}}\left(500 \mathrm{MHz} ; \mathrm{CD}_{2} \mathrm{Cl}_{2}\right) 11.97(2 \mathrm{H}$, br s, NH), 7.60-7.52 (10H, m, $\operatorname{Ar} \mathbf{H}), 7.34-7.31(2 \mathrm{H}, \mathrm{m}, \operatorname{Ar} \mathbf{H})$, $7.25(4 \mathrm{H}, \mathrm{m}, \operatorname{Ar} \mathbf{H}), 7.09(1 \mathrm{H}, \mathrm{s}, \mathbf{H}-13), 6.72(2 \mathrm{H}, \mathrm{d}, J 8.5 \mathrm{~Hz}, \operatorname{Ar} \mathbf{H}), 5.03(1 \mathrm{H}, \mathrm{s}, \mathbf{H}-6)$; $\delta_{\mathrm{C}}\left(125 \mathrm{MHz} ; \mathrm{CD}_{3} \mathrm{CN}\right) 146.15,143.48,136.34,133.38,132.99,132.82,131.73,131.18,131.02$, 127.78, 119.86, 119.84, 98.06 (C-6), 95.37 (C-13); m/z (FAB) $437\left(\mathrm{M}^{+}-\mathrm{H} .2 \mathrm{ClO}_{4}, 55 \%\right.$ ) (Found: $\mathrm{M}^{+}-\mathrm{H} .2 \mathrm{ClO}_{4}, 437.1767 . \mathrm{C}_{30} \mathrm{H}_{21} \mathrm{~N}_{4}$ requires $\left.\mathrm{M}-\mathrm{H} .2 \mathrm{ClO}_{4}, 437.1766\right)$.

\section{Acknowledgements}

I thank Prof. F. Wudl for financial (National Science Foundation Grant DMR9812046) and intellectual support during this work, which was carried out in Prof F. Wudl's laboratories at the Exotic Materials Institute, University of California, Los Angeles.

\section{References}

1. Wudl, F.; Koutentis, P. A.; Weitz, A.; Ma, B.; Strassner, T.; Houk, K. N.; Khan S.I. Pure \& Appl. Chem. 1999, 71, 295.

2. Choi, H.; Yang, X.; Mitchell, G. W.; Collier, C. P.; Wudl, F.; Heath, J. R. J. Phys. Chem. B 2002, 106, 1833. 
3. Riley, A. E.; Mitchell, G. W.; Koutentis, P. A.; Bendikov, M.; Kaszynki, P.; Wudl F.; Tolbert, S. H. Adv. Funct. Mater. accepted.

4. Armand, J.; Boulares, L.; Bellec, C.; Pinson, J. Can. J. Chem. 1987, 65, 1619.

5. (a) Kehrmann, F.; Bürgin, H. Chem. Ber. 1896, 29, 1820. (b) Kehrmann, F.; Duret, A. Chem. Ber. 1898, 31, 2442. (c) Cassella \& Co., D.R.P. 142 565; C. 1903 II, 85; Frdl. 7, 345.

6. (a) Witt, O. N.; Chem. Ber. 1887, 20, 1538. (b) Fischer, O.; Hepp, E. Chem. Ber. 1890, 23, 789. (c) Fischer, O.; Hepp, E. Chem. Ber. 1895, 28, 293. (d) Kehrmann, F.; Bürgin, H. Chem. Ber. 1896, 29, 1246.

7. Barry, V. C.; Belton, J. G.; O'Sullivan, J. F.; Twomey, D. J. Chem. Soc. 1956, 888.

8. Kulagowski, J. J.; Rees, C. W. Synthesis 1980, 215.

9. Nietzki, R.; Slaboszewicz, J. Chem. Ber. 1901, 34, 3727. 